\title{
Hot corinos in NGC 1333-IRAS4B and IRAS2A *
}

\author{
S. Bottinelli ${ }^{1,2}$, C. Ceccarelli ${ }^{1}$, J. P. Williams ${ }^{2}$, and B. Lefloch ${ }^{1}$ \\ ${ }^{1}$ Laboratoire d'Astrophysique de l'Observatoire de Grenoble, BP 53, 38041 Grenoble Cedex 9, France \\ e-mail: [sbottine, ceccarel, lefloch]@obs.ujf-grenoble.fr \\ 2 Institute for Astronomy, University of Hawai 'i, 2680 Woodlawn Drive, Honolulu HI 96822, USA \\ e-mail: jpw@ifa.hawaii.edu \\ Received 3 March 2006 / Accepted 11 October 2006
}

\section{ABSTRACT}

\begin{abstract}
Context. Complex organic molecules have been detected in massive hot cores for over two decades, and only recently in three hot corinos (the inner regions surrounding Sun-like protostars, where the dust temperature exceeds $100 \mathrm{~K}$ ). Since hot corinos have sizes of $\sim 100 \mathrm{AU}$ (i.e., of the order of the extent of the Solar System), it is particularly relevant to understand whether they are common and to identify the formation route(s) of complex organic molecules. Much has yet to be learned on this topic, since even recent models predicted it was not possible to form these molecules in low-mass protostars.

Aims. We aim to enlarge the number of known hot corinos and carry out a first comparative study with hot cores. The ultimate goal is to understand whether complex organic molecules form in the gas phase or on grain surfaces, and what the possible key parameters are. Methods. We observed millimeter rotational transitions of $\mathrm{HCOOH}, \mathrm{HCOOCH}_{3} \mathrm{CH}_{3} \mathrm{OCH}_{3} \mathrm{CH}_{3} \mathrm{CN}$ and $\mathrm{C}_{2} \mathrm{H}_{5} \mathrm{CN}$ in a sample of low-mass protostars with the IRAM-30 m. Using the rotational diagram method coupled with the information about the sources' structure, we calculate the abundances of the observed molecules. To interpret these abundances, we review the proposed formation processes of the above molecules.

Results. We report the detection of $\mathrm{HCOOCH}_{3}$ and/or $\mathrm{CH}_{3} \mathrm{CN}$ towards NGC 1333-IRAS4B and NGC 1333-IRAS2A. We find that abundance ratios of O-bearing molecules to methanol or formaldehyde in hot corinos are comparable and about unity, and are relatively (depending on how the ratios are determined) higher than those in hot cores and in Galactic center clouds.

Conclusions. So far, complex organic molecules were detected in all the hot corinos where they were searched for, suggesting that it is a common phase for low-mass protostars. While some evidence points to grain-surface synthesis (either in the cold or warm-up phase) of these molecules (in particular for $\mathrm{HCOOH}$ and $\mathrm{HCOOCH}_{3}$ ), the present data do not allow us to disregard gas-phase formation. More observational, laboratory, and theoretical studies are required to improve our understanding of hot corinos.
\end{abstract}

Key words. ISM: abundances - ISM: molecules - stars: formation

\section{Introduction}

Many aspects of the formation of solar-type stars have now been elucidated and, even though the details are much debated, there exists a widely accepted framework for it (see the volume Protostars and Planets V, for example, Ceccarelli et al. 2006; Ward-Thompson et al. 2006; White et al. 2006). Low-mass stars form inside molecular clouds from dense and cold condensations, called prestellar cores, which evolve into Class 0 and then Class I sources. In the latest phases, the newly born star is surrounded by a proto-planetary disk, which eventually may form planets.

Somewhat less understood, and therefore more frequently debated, is the chemical evolution of matter from the molecular cloud to the proto-planetary phase, and then to planets (e.g., Ceccarelli et al. 2006). It is now acknowledged that, at the densities and temperatures typical of the centers of prestellar cores $\left(\geq 10^{6} \mathrm{~cm}^{-3}\right.$ and $\leq 10 \mathrm{~K}$, respectively), heavy-element bearing molecules condense out onto dust grains, forming icy mantles. Very likely, hydrogenation and oxidation of small molecules and atoms (like $\mathrm{CO}, \mathrm{O}, \mathrm{N}$, etc.) occur on the grain surfaces, so that the mantles end up being composed mainly of water, interspersed with traces of formaldehyde, methanol, ammonia, and possibly even more complex molecules. As material from the surrounding envelope starts accreting onto the central protostar,

* Appendix is only available in electronic form at http://www . aanda.org the increased radiation output heats up the surroundings. When and where the dust reaches the appropriate sublimation temperature of the ices (which depends on the exact composition of these ices), the grain mantles evaporate, injecting their components into the gas phase. These components can further react to form more complex molecules. The regions where the ice mantles sublimate (at $T_{\text {dust }} \gtrsim 100 \mathrm{~K}$ ) and where the emission from complex molecules (whether evaporated from the grain mantles or formed in the gas) originates, are called hot corinos (Ceccarelli 2004; Bottinelli et al. 2004a). The chemical composition of these hot corinos reflects both the heritage from the prestellar core phase and the reactions taking place in the warm gas. The result is a gas rich in complex organic molecules (Cazaux et al. 2003), whose diversity is far from being fully explored and understood. It is likely that we have discovered only the tip of the iceberg. Moreover, the story does not end here. Indeed, the fate of these complex organic molecules is almost totally unknown. They may condense onto the grain surfaces again during the proto-planetary disk phase. Perhaps they are incorporated into the planetesimals forming the building blocks of planets, or into comets and asteroids, in which case they may end up on newly formed planets as accretion proceeds. This picture is, at least partially, supported by the fact that some complex organic molecules are found both in hot corinos and in comets (e.g., methyl cyanide $\mathrm{CH}_{3} \mathrm{CN}$, methyl formate $\mathrm{HCOOCH}_{3}$, and formic acid $\mathrm{HCOOH}$; Ehrenfreund \& Charnley 2000). For these 
reasons, the hot corino phase is not only interesting in itself, but it is a critical phase in the process of solar-type star formation.

In short, the molecular complexity in hot corinos is particularly relevant to molecular astrophysics and (exo)planetary science, but particularly unknown. Our ignorance can be summarized by this simple, multiple-part question: which complex organic molecules are formed, and why, where, and how does this take place? At present we are simply unable to answer this question for the following reasons. (i) We do not have a census of the complex organic molecules. We suspect that many complex molecules are present in the millimeter spectra of hot corinos, but the firm identification of a large molecule requires several lines from this molecule and can be hampered by the multitude of weak lines in the spectra (e.g., Combes et al. 1996; Ceccarelli et al. 2000c). (ii) We do not know why they form. The gas-grain models for hot cores (the high-mass analogs of hot corinos) predict that there is not enough time for hot corinos to form molecules because the gas falls rapidly towards the central forming star before any gas-phase reaction can lead to complex organic molecules (e.g., Schöier et al. 2002). (iii) We debate whether the observed complex molecules reside in the passive heated envelope (Ceccarelli et al. 2000a,b), in the hidden circumstellar disk (Jørgensen et al. 2005b), or in the interface between the outflow and the envelope (Chandler et al. 2005). (iv) Finally, we do not know how complex molecules form: in the gas-phase or on the grain surfaces, or both. Much of our ignorance stems from the too few hot corinos so far studied: IRAS 16293-2422 (hereafter IRAS 16293, Cazaux et al. 2003; Kuan et al. 2004; Bottinelli et al. 2004b; Remijan \& Hollis 2006), NGC 1333-IRAS4A (hereafter IRAS4A, Bottinelli et al. 2004a), NGC 1333-IRAS4B (hereafter IRAS4B, Sakai et al. 2006), and NGC 1333-IRAS2A (hereafter IRAS2A, Jørgensen et al. 2005a).

In this paper, we present detections of complex organic molecules in two Class 0 hot corinos: $\mathrm{HCOOCH}_{3}$ and $\mathrm{CH}_{3} \mathrm{CN}$ in IRAS4B, and $\mathrm{CH}_{3} \mathrm{CN}$ in IRAS2A. The article is organized as follows: details on the choice of sources are given in Sect. 2, the observations are described in Sect. 3 and results are presented and discussed in Sects. 4 and 5, respectively. Section 5 starts with some remarks on the formation routes of complex organic molecules (Sect. 5.1, based on a summary of the possible formation mechanisms of the complex molecules $\mathrm{HCOOH}, \mathrm{HCOOCH}_{3}, \mathrm{CH}_{3} \mathrm{OCH}_{3}, \mathrm{CH}_{3} \mathrm{CN}$, and $\mathrm{C}_{2} \mathrm{H}_{5} \mathrm{CN}$ given in Appendix A), followed by the analysis of our data (Sects. 5.2 and 5.3), and ends with a comparison of hot corinos with hot cores (Sect. 5.4) and with Galactic center clouds (Sect. 5.5). Finally, a summary and concluding remarks are given in Sect. 6.

\section{Source selection and background}

We observed two Class 0 protostars, IRAS4B and IRAS2A, located in the Perseus complex, specifically in the NGC 1333 cloud, whose distance is estimated to be 220 pc (Černis 1990). IRAS4B belongs to the multiple system IRAS4 and is located $\sim 30^{\prime \prime}$ and $\sim 17^{\prime \prime}$ from the other two components, IRAS4A and IRAS4C (Looney et al. 2000; Reipurth et al. 2002). It may be a multiple stellar system itself (Looney et al. 2000). Both IRAS4A and IRAS4B are associated with molecular outflows of a dynamical age of a few thousand years, seen in CO, CS (Blake et al. 1995), and $\mathrm{SiO}$ (Lefloch et al. 1998). IRAS2A is part of a protobinary system and is separated by $30^{\prime \prime}$ from its companion IRAS2B (Looney et al. 2000; Reipurth et al. 2002). Two CO bipolar outflows appear to originate within a few arcsec of IRAS2A: a highly collimated jet in the east-west direction and a large-scale outflow aligned NNE-SSW (Sandell et al. 1994; Knee \& Sandell 2000).

IRAS4B and IRAS2A were selected from the sample studied in Maret et al. $(2004,2005)$ because they both are good hot corino candidates and because, due to their distance and luminosity, they are expected to have brighter lines compared to other Class 0 sources (e.g., from the André et al. 2000 sample). The candidacy relies upon the claim by Maret et al. (2004, 2005) of the presence, in both IRAS2A and IRAS4B, of a warm $(\gtrsim 100 \mathrm{~K})$ inner region where grain mantles sublimate. The claim is based on the observed jumps in the abundances of formaldehyde and methanol: low abundances in the outer, cold envelope where formaldehyde and methanol are still frozen onto grain surfaces, and high abundances in the inner, warm envelope where the heat from the central object causes the desorption of formaldehyde and methanol into the gas phase. Indeed, in the two sources where hot corinos have been detected so far, IRAS 16293 (Cazaux et al. 2003) and IRAS4A (Bottinelli et al. 2004a), similar jumps in the formaldehyde and/or methanol abundances have also been claimed (Maret et al. 2004, 2005), supporting the choice of our targets.

However, there is a noticeable difference in the extent of the hot corinos (54 to $266 \mathrm{AU}$, Maret et al. 2004) and in the jump sizes in all four sources. Indeed, according to Maret et al. (2005), methanol abundances show jumps of a factor 100 to 350 in all sources but IRAS4A, where the jump is lower than about a factor 15, whereas Maret et al. (2004) found formaldehyde abundance jumps of a factor 100 to 6000. Other authors have carried out analyses of methanol and formaldehyde in low-mass protostars (e.g., Schöier et al. 2002; Jørgensen et al. 2005b). In contrast with what was found by Maret et al. (2004), Jørgensen et al. (2005b) claimed that no jump of formaldehyde abundance is required to model the line intensities in any of the sources except IRAS 16293 (Schöier et al. 2002). However, like Maret et al. (2005), Jørgensen et al. (2005b) and Schöier et al. (2002) found a methanol abundance jump in IRAS4B, IRAS2A, and IRAS 16293, but not necessarily in IRAS4A. The hot corino abundances of formaldehyde and methanol found by the two groups are summarized in Table 1. In the following we will adopt the Maret et al. $(2004,2005)$ framework, but we will also discuss the results in the light of the Jørgensen et al. (2005b) analysis.

In summary, the four hot corinos - IRAS 16293, IRAS4A, IRAS4B, and IRAS2A - form a sample of interestingly different sources, both because they span an apparent large range of formaldehyde and methanol abundances, and because the very existence of the abundance jumps is still debated. Hence, even though statistically small, this sample will permit a first assessment of how the presence and abundance of complex organic molecules in hot corinos depend on formaldehyde and methanol. Indeed, these two molecules are predicted to be among the most important parent ones (e.g., Caselli et al. 1993; Rodgers \& Charnley 2001, 2003), but it is not always clear how they relate to the complex molecules (e.g., Horn et al. 2004).

\section{Observations}

The observations were carried out in June 2003 with the 30-m telescope of the Institut de RadioAstronomie Millimétrique (IRAM) $)^{1}$. The positions used for pointing were $(\alpha, \delta)(2000)=\left(03^{\mathrm{h}} 29^{\mathrm{m}} 12^{\mathrm{s}} .0,31^{\circ} 13^{\prime} 09^{\prime \prime}\right)$ for IRAS4B, and $(\alpha$, $\delta)(2000)=\left(03^{\mathrm{h}} 28^{\mathrm{m}} 55^{\mathrm{s}} .4,31^{\circ} 14^{\prime} 35^{\prime \prime}\right)$ for IRAS2A. Based on the

1 IRAM is an international venture supported by INSU/CNRS (France), MPG (Germany), and IGN (Spain). 
Table 1. Hot corino abundances for formaldehyde $\left(X_{\mathrm{hc}}\left(\mathrm{H}_{2} \mathrm{CO}\right)\right)$ and methanol $\left(\mathrm{X}_{\mathrm{hc}}\left(\mathrm{CH}_{3} \mathrm{OH}\right)\right)$.

\begin{tabular}{|c|c|c|c|c|c|}
\hline \multirow[t]{2}{*}{ Source } & \multirow{2}{*}{$\begin{array}{r}L_{\mathrm{bol}}{ }^{a} \\
\left(L_{\odot}\right)\end{array}$} & \multicolumn{2}{|c|}{$X_{\mathrm{hc}}\left(\mathrm{H}_{2} \mathrm{CO}\right)$} & \multicolumn{2}{|c|}{$X_{\mathrm{hc}}\left(\mathrm{CH}_{3} \mathrm{OH}\right)$} \\
\hline & & Maret et al. (2004) & Jørgensen et al. $(2005 b)^{b}$ & Maret et al. (2005) & Jørgensen et al. $(2005 b)^{c}$ \\
\hline IRAS 16293-2422 & 27 & $1 \times 10^{-7}$ & $6 \times 10^{-8 d}$ & $1 \times 10^{-7}$ & $1 \times 10^{-7}$ \\
\hline NGC 1333-IRAS4A & 6 & $2 \times 10^{-8}$ & $3 \times 10^{-9}$ & $<1 \times 10^{-8}$ & $\leq 3.5 \times 10^{-9}$ \\
\hline NGC 1333-IRAS4B & 6 & $3 \times 10^{-6}$ & $1 \times 10^{-8}$ & $7 \times 10^{-7}$ & $9.5 \times 10^{-8}$ \\
\hline NGC 1333-IRAS2A & 16 & $2 \times 10^{-7}$ & $8 \times 10^{-10}$ & $3 \times 10^{-7}$ & $1.5 \times 10^{-7}$ \\
\hline
\end{tabular}

${ }^{a}$ From Jørgensen et al. (2002).

${ }^{b}$ Note that except in IRAS 16293 (Schöier et al. 2002), the formaldehyde abundances modeled by Jørgensen et al. (2005b) do not require any jump (see text for further details).

${ }^{c}$ Methanol abundances averaged over A- and E-types.

${ }^{d}$ From Schöier et al. (2002).

Table 2. Observed frequencies and targeted molecules.

\begin{tabular}{cllcc}
\hline \hline $\begin{array}{c}\text { Frequency range } \\
(\mathrm{GHz})\end{array}$ & Molecule & $\begin{array}{l}\text { Frequencies } \\
(\mathrm{GHz})\end{array}$ & $\begin{array}{c}\text { rms (mK) } \\
\text { IRAS4B }\end{array}$ & $\begin{array}{c}\text { rms (mK) } \\
\text { IRAS2A }\end{array}$ \\
\hline $90.07-90.32$ & $\mathrm{HCOOCH}_{3}-\mathrm{A}$ & $90.156,90.229$ & 7 & 2 \\
& $\mathrm{HCOOCH}_{3}-\mathrm{E}$ & $90.145,90.227$ & 2,7 & 2 \\
& $\mathrm{HCOOH}$ & 90.164 & 2 & 2 \\
$98.50-98.75$ & $\mathrm{HCOOCH}_{3}-\mathrm{A}$ & $98.611,98.682$ & 7,2 & 3 \\
& $\mathrm{HCOOCH}_{3}-\mathrm{E}$ & $98.606,98.711$ & 7,2 & 3 \\
& $\mathrm{C}_{2} \mathrm{H}_{5} \mathrm{CN}$ & $98.610,98.701$ & 7,2 & 3 \\
$110.29-110.43$ & $\mathrm{CH}_{3} \mathrm{CN}$ & $110.329-110.383$ & 5 & - \\
$223.17-223.42$ & $\mathrm{CH}_{3} \mathrm{OCH}$ & $223.200-223.204$ & 13 & 34 \\
& $\mathrm{C}_{2} \mathrm{H}_{5} \mathrm{CN}_{3}$ & 223.385 & 13 & 34 \\
$226.50-227.00$ & $\mathrm{HCOOCH}_{3}-\mathrm{A}$ & $226.718,226.778$ & 11 & 15 \\
& $\mathrm{HCOOCH}_{3}-\mathrm{E}$ & $226.713,226.773$ & 11 & 15 \\
$257.35-257.55$ & $\mathrm{CH}_{3} \mathrm{CN}$ & $257.403-257.527$ & 22 & 28 \\
\hline
\end{tabular}

Note. A dash indicates that no data were taken in the corresponding frequency range.

observations of IRAS 16293 by Cazaux et al. (2003), we targeted the following complex molecules: methyl formate, $\mathrm{HCOOCH}_{3}$ (A and E), formic acid, $\mathrm{HCOOH}$, dimethyl ether, $\mathrm{CH}_{3} \mathrm{OCH}_{3}$, methyl cyanide, $\mathrm{CH}_{3} \mathrm{CN}$, and ethyl cyanide, $\mathrm{C}_{2} \mathrm{H}_{5} \mathrm{CN}$. Different telescope settings were used to include as many transitions as possible for each molecule (see Table 2). All lines were observed with a low resolution, $1 \mathrm{MHz}$ filter bank of $4 \times 256$ channels split between different receivers, providing a velocity resolution of $\sim 3,2$, and $1 \mathrm{~km} \mathrm{~s}^{-1}$ at 3,2 , and $1 \mathrm{~mm}$, respectively. Each receiver was simultaneously connected to a unit of the autocorrelator, with spectral resolutions of 20,80 , or $320 \mathrm{kHz}$ and bandwidths between 40 and $240 \mathrm{MHz}$, equivalent to an (unsmoothed) velocity resolution of $0.1-0.4 \mathrm{~km} \mathrm{~s}^{-1}$ at 3,2 , and $1 \mathrm{~mm}$. Typical system temperatures were $100-200 \mathrm{~K}$, $180-250 \mathrm{~K}$, and $500-1500 \mathrm{~K}$, at 3,2 , and $1 \mathrm{~mm}$, respectively.

Two observation modes were used: position switching with the OFF position at an offset of $\Delta \alpha=-100^{\prime \prime}, \Delta \delta=+300^{\prime \prime}$, and wobbler switching with a $110^{\prime \prime}$ throw in azimuth. Pointing and focus were regularly checked using planets or strong quasars, providing a pointing accuracy of $3^{\prime \prime}$. All intensities reported in this paper are expressed in units of main-beam brightness temperature. At 3, 2, and $1 \mathrm{~mm}$, the angular resolution is 24,16 , and $10^{\prime \prime}$ and the main beam efficiency is 76,69 , and $50 \%$, respectively. Figure 1 shows the obtained spectra.

\section{Results}

Detected transitions have been identified using the JPL molecular line catalog (Pickett et al. 1998), the Cologne Database for Molecular Spectroscopy (Müller et al. 2001, 2005), and the National Institute of Standards and Technology (NIST)
Recommended Rest Frequencies for Observed Interstellar Molecular Microwave Transitions (Lovas \& Dragoset 2004). They are reported in Table 3 . We considered only lines with a $3-\sigma$ detection and a $V_{\mathrm{LSR}}=7.0 \pm 0.4 \mathrm{~km} \mathrm{~s}^{-1}$ as good identifications. We detected two of the five targeted molecules: 4 transitions for $\mathrm{HCOOCH}_{3}-\mathrm{A}$ in IRAS4B and 5 and 6 transitions for $\mathrm{CH}_{3} \mathrm{CN}$ in IRAS4B and IRAS2A, respectively. We also have a possible detection for $\mathrm{C}_{2} \mathrm{H}_{5} \mathrm{OH}$ at $226.661 \mathrm{GHz}$ in IRAS4B ${ }^{2}$. Note that no observations were made at $110 \mathrm{GHz}$ for IRAS2A (see Table 2), and that the rms reached at $257 \mathrm{GHz}$ for IRAS4B is too high to detect the $\mathrm{CH}_{3} \mathrm{CN}$ transitions at this frequency, if the line ratios are similar to those in IRAS4A.

To derive rotational temperatures and beam-averaged column densities (see Table 4), we used the rotational diagram method (Fig. 2). For this analysis, we assumed that the emission from a given molecule is unresolved in the smallest beam in which a transition of that molecule was detected $\left(\theta_{\min }\right.$, which is always $\geq 10^{\prime \prime}-$ see Cols. 3 and 7 in Table 4). This is justified by the interferometric observations of IRAS 16293-2422 (Bottinelli et al. 2004b; Kuan et al. 2004; Chandler et al. 2005), NGC 1333-IRAS2A (Jørgensen et al. 2005a), and NGC 1333-IRAS4A (Bottinelli et al. 2006), which show that the bulk of the emission occurs over a region $\lesssim 1^{\prime \prime}$. We therefore corrected the integrated intensities observed at lower frequencies for beam dilution with respect to $\theta_{\min }$. Then, the hot corino abundances were obtained by scaling the beamaveraged column densities to the sizes of the hot corinos (taken

\footnotetext{
2 This transition is not seen in NGC 1333-IRAS4A. In this source, there is a tentative detection at $90.118 \mathrm{GHz}$ (Bottinelli et al. 2004a) that has a lower energy $\left(3.5\right.$ vs. $\left.28 \mathrm{~cm}^{-1}\right)$, but also lower line strength $(\log (I)=-4.8$ vs. -3.9$)$.
} 

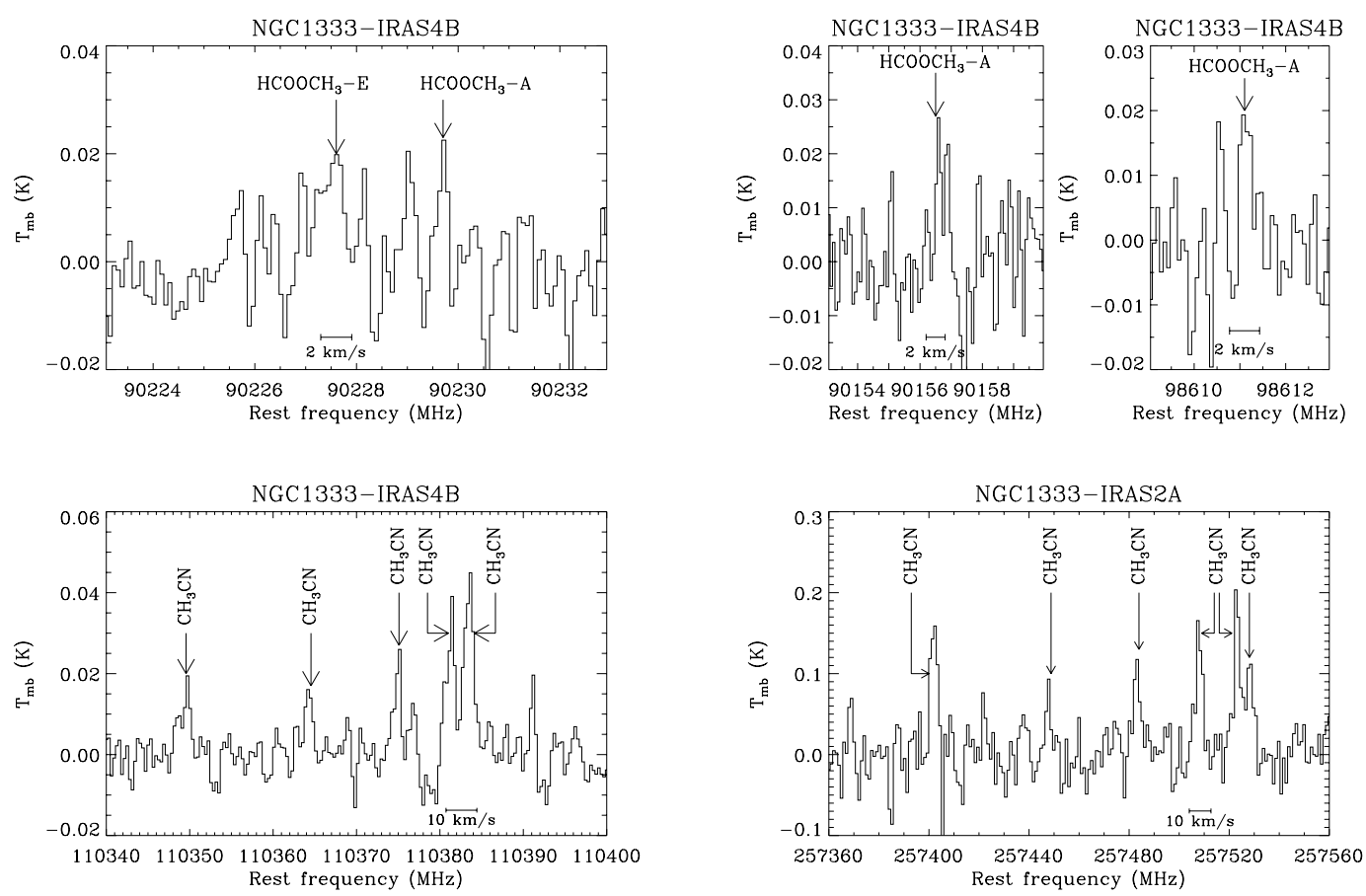

Fig. 1. Observed spectra towards IRAS4B and IRAS2A. The rms are $7 \mathrm{mK}$ (top panels) and 5 and $28 \mathrm{mK}$ (bottom panels, left to right); the spectral resolutions are $0.2,0.3,0.8$, and $1.2 \mathrm{~km} \mathrm{~s}^{-1}$ at $90,98,110$, and $257 \mathrm{GHz}$, respectively. Detected transitions are (from left to right, top to bottom): $\mathrm{HCOOCH}_{3}-\mathrm{E}\left(8_{0,8}-7_{0,7}\right), \mathrm{HCOOCH}_{3}-\mathrm{A}\left(8_{0,8}-7_{0,7}, 7_{2,5}-6_{2,4}, 8_{3,6}-7_{3,5}\right), \mathrm{CH}_{3} \mathrm{CN}\left(6_{K}-5_{K}, K=4, \ldots, 0\right)$, and $\mathrm{CH}_{3} \mathrm{CN}\left(14_{K}-13_{K}, K=5, \ldots, 0\right)$. Unlabeled lines are unidentified.

Table 3. Molecular lines detected.

\begin{tabular}{lcccccc}
\hline \hline Molecule & Transition line & $\begin{array}{c}\text { Frequency } \\
(\mathrm{MHz})\end{array}$ & $\begin{array}{c}E_{u}{ }^{a} \\
\left(\mathrm{~cm}^{-1}\right)\end{array}$ & $\begin{array}{c}T_{\mathrm{mb}} \\
(\mathrm{mK})\end{array}$ & $\begin{array}{c}\Delta V^{b} \\
\left(\mathrm{~km} \mathrm{~s}^{-1}\right)\end{array}$ & $\begin{array}{c}\int T_{\mathrm{mb}} \mathrm{d} V \\
\left(\mathrm{~K} \mathrm{~km} \mathrm{~s}^{-1}\right)\end{array}$ \\
\hline $\mathrm{HCOOCH}_{3}$-A & $7_{2,5}-6_{2,4}$ & 90156.5 & 13.7 & 26 & 0.6 & $0.017 \pm 0.004$ \\
& $8_{0,8}-7_{0,7}$ & 90229.7 & 13.9 & 23 & 0.5 & $0.013 \pm 0.004$ \\
& $8_{3,6}-7_{3,5}$ & 98611.1 & 18.9 & 21 & 0.7 & $0.016 \pm 0.004$ \\
$\mathrm{HCOOCH}_{3}-\mathrm{E}$ & $8_{0,8}-7_{0,7}$ & 90227.8 & 14.0 & 18 & $1.8^{d}$ & $0.035 \pm 0.005$ \\
$\mathrm{CH}_{3} \mathrm{CN}^{c}$ & $6_{4,0}-5_{4,0}$ & 110349.7 & 92.3 & 18 & 2.3 & $0.045 \pm 0.009$ \\
& $6_{3,0}-5_{3,0}$ & 110364.6 & 57.6 & 17 & 2.8 & $0.049 \pm 0.011$ \\
& $6_{2,0}-5_{2,0}$ & 110375.1 & 32.8 & 24 & 2.5 & $0.062 \pm 0.013$ \\
& $6_{1,0}-5_{1,0}$ & 110381.5 & 17.9 & 33 & 3.4 & $0.119 \pm 0.014$ \\
& $6_{0,0}-5_{0,0}$ & 110383.6 & 12.9 & 42 & 3.8 & $0.171 \pm 0.015$ \\
\hline $\mathrm{CH}_{3} \mathrm{CN}^{c}$ & $14_{5,0}-13_{5,0}$ & 257403.6 & 188.5 & 169 & 3.8 & $0.692 \pm 0.091$ \\
& $14_{4,0}-13_{4,0}$ & 257448.9 & 143.9 & 111 & 1.6 & $0.190 \pm 0.046$ \\
& $14_{3,0}-13_{3,0}$ & 257482.7 & 109.1 & 113 & 3.4 & $0.413 \pm 0.044$ \\
& $14_{2,0}-13_{2,0}$ & 257507.9 & 84.3 & 145 & 2.7 & $0.411 \pm 0.055$ \\
& $14_{1,0}-13_{1,0}$ & 257522.5 & 69.4 & 198 & 3.1 & $0.662 \pm 0.087$ \\
& $14_{0,0}-13_{0,0}$ & 257527.4 & 64.4 & 115 & 3.8 & $0.470 \pm 0.068$ \\
\hline
\end{tabular}

${ }^{a}$ Energy of the upper level of the transition.

${ }^{b}$ Width of the observed line.

${ }^{c}$ All the $\mathrm{CH}_{3} \mathrm{CN}$ lines are unresolved triplets, except at $110349.7 \mathrm{MHz}$, which is an unresolved doublet. The quoted signal is the integral over each triplet or doublet.

${ }^{d}$ This line of the $\mathrm{E}$ form of $\mathrm{HCOOCH}_{3}$ has a width about three times as large as that of the corresponding transition of the A form, which could be due to the presence of some unknown transition(s) artificially increasing the width.

from Table 6 of Maret et al. 2004: 0.'25 for IRAS4B and 0.'43 for IRAS2A) and dividing by the hot corino $\mathrm{H}_{2}$ column density. The latter was obtained from the density profiles derived by Jørgensen et al. (2002), based on single-dish observations of the dust continuum and radiative transfer calculations (see also Table 6 of Maret et al. 2004). This introduces an uncertainty in the derived abundances, due to the uncertain sizes and
$\mathrm{H}_{2}$ column densities of the hot corinos, caused by the extrapolation from single-dish observations. However, abundance ratios are not affected by this uncertainty, unless different molecules are formed in different regions (see discussion in Sect. 5.4).

We make the following remarks about the results we obtain. The rotational temperature of $\mathrm{HCOOCH}_{3}-\mathrm{A}$ is poorly constrained due to the absence of points at higher energies. More 
Table 4. Results from the rotational diagrams and upper limits for IRAS4B and IRAS2A.

\begin{tabular}{|c|c|c|c|c|c|c|c|c|}
\hline \multirow[b]{2}{*}{ Molecule } & \multicolumn{4}{|c|}{ IRAS4B } & \multicolumn{4}{|c|}{ IRAS2A } \\
\hline & $\begin{array}{l}T_{\text {rot }} \\
(\mathrm{K}) \\
\end{array}$ & $\begin{array}{c}\theta_{\min } \\
\left({ }^{\prime \prime}\right) \\
\end{array}$ & $\begin{array}{l}N_{\text {beam }}{ }^{b} \\
\left(\mathrm{~cm}^{-2}\right)\end{array}$ & $X_{\mathrm{hc}}{ }^{c}$ & $\begin{array}{l}T_{\text {rot }} \\
(\mathrm{K}) \\
\end{array}$ & $\begin{array}{c}\theta_{\min } \\
\left({ }^{\prime \prime}\right) \\
\end{array}$ & $\begin{array}{l}N_{\text {beam }}{ }^{b} \\
\left(\mathrm{~cm}^{-2}\right) \\
\end{array}$ & $X_{\mathrm{hc}}{ }^{d}$ \\
\hline $\mathrm{HCOOCH}_{3}-\mathrm{A}$ & $38_{-35}^{+49}$ & 10 & $(4.7 \pm 3.6) \times 10^{13}$ & $(1.1 \pm 0.8) \times 10^{-6}$ & [38] & 10 & $<2.9 \times 10^{14}$ & $<6.7 \times 10^{-7}$ \\
\hline $\mathrm{HCOOH}$ & {$[38]$} & 22 & $<1.0 \times 10^{13}$ & $<1.0 \times 10^{-6}$ & [38] & 25 & $<7.4 \times 10^{12}$ & $<1.2 \times 10^{-7}$ \\
\hline $\mathrm{CH}_{3} \mathrm{OCH}_{3}$ & {$[38]$} & 10 & $<6.8 \times 10^{13}$ & $<1.2 \times 10^{-6}$ & [38] & 10 & $<1.8 \times 10^{14}$ & $<4.2 \times 10^{-7}$ \\
\hline $\mathrm{CH}_{3} \mathrm{CN}$ & $39 \pm 3$ & 22 & $(9.7 \pm 1.0) \times 10^{11}$ & $(9.5 \pm 0.2) \times 10^{-8}$ & $87 \pm 17$ & 10 & $(3.7 \pm 1.0) \times 10^{12}$ & $(8.7 \pm 2.4) \times 10^{-9}$ \\
\hline $\mathrm{C}_{2} \mathrm{H}_{5} \mathrm{CN}$ & [39] & 10 & $<4.2 \times 10^{13}$ & $<7.5 \times 10^{-7}$ & [87] & 10 & $<4.3 \times 10^{13}$ & $<1.0 \times 10^{-7}$ \\
\hline
\end{tabular}

Note - When they could not be derived from a rotational diagram, temperatures have been assumed to be equal to that of $\mathrm{HCOOCH}_{3}$ and $\mathrm{CH}_{3} \mathrm{CN}$ for O-bearing and N-bearing molecules, respectively. In this case, the values are shown in square brackets.

${ }^{a}$ Smallest beam size for which a transition was detected (see text for details).

${ }^{b}$ Column density averaged over $\theta_{\min }$.

${ }^{c}$ Abundance in the hot corino assuming an $\mathrm{H}_{2}$ column density of $N\left(\mathrm{H}_{2}\right)=8.1 \times 10^{22} \mathrm{~cm}^{-2}$ and a hot corino size of $54 \mathrm{AU}$, i.e., 0.25 " at $220 \mathrm{pc}$ (from Maret et al. 2004).

${ }^{d}$ Abundance in the hot corino assuming an $\mathrm{H}_{2}$ column density of $N\left(\mathrm{H}_{2}\right)=2.1 \times 10^{23} \mathrm{~cm}^{-2}$ and a hot corino size of 94 AU, i.e., $0.43^{\prime \prime}$ at 220 pc (from Maret et al. 2004).
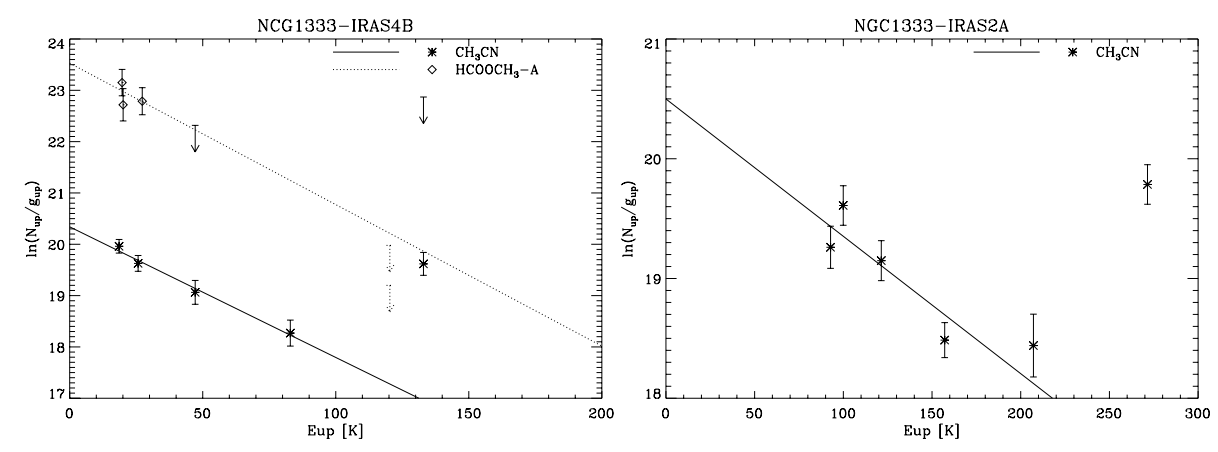

Fig. 2. Rotational diagrams of the detected molecules, corrected for beam dilution at lower frequencies. The arrows show the upper limits for undetected transitions. Lines represent the best fit to the data. Error bars are derived assuming a calibration uncertainty of $10 \%$ on top of the statistical error. The excess of emission of the $\mathrm{CH}_{3} \mathrm{CN}$ transition at $270 \mathrm{~K}$ in IRAS2A and $135 \mathrm{~K}$ in IRAS4B is probably due to contamination from $\mathrm{CH}_{3} \mathrm{OH} 18_{3,16}-18_{2,17}$ and unknown line(s), respectively, and are not included in the fits. generally, some of the rotational temperatures are below $100 \mathrm{~K}$. This could be due to non-LTE and/or line opacity effects. We find that the derived $\mathrm{CH}_{3} \mathrm{CN}$ abundance is consistent with the value obtained by Jørgensen et al. (2005b) for an inner $(T>90 \mathrm{~K})$ region. Upper limits were calculated for the targeted molecules that were not detected: $\mathrm{HCOOH}$ and $\mathrm{C}_{2} \mathrm{H}_{5} \mathrm{CN}$ in both sources, $\mathrm{HCOOCH}_{3}-\mathrm{A}$ in IRAS2A, and $\mathrm{CH}_{3} \mathrm{OCH}_{3}$ in IRAS4B. The upper limits for the O-bearing molecules were derived using the rotational temperature derived for $\mathrm{HCOOCH}_{3}-\mathrm{A}$ in IRAS4B, whereas the upper limits for $\mathrm{N}$-bearing molecules were calculated assuming a rotational temperature equal to that of $\mathrm{CH}_{3} \mathrm{CN}$ in each source. Finally, the $\mathrm{CH}_{3} \mathrm{OCH}_{3}$ abundance in IRAS2A was taken from Jørgensen et al. (2005a): the quoted abundance ( $3 \times 10^{-8}$ in an inner, $T>90 \mathrm{~K}$ region) is consistent with the upper limit derived from our data $\left(x<4.2 \times 10^{-7}\right.$, also calculated using the rotational temperature derived for $\mathrm{HCOOCH}_{3}-\mathrm{A}$ in IRAS4B). Jørgensen et al. (2005a) also report a tentative detection of $\mathrm{HCOOCH}_{3}$, but do not give an estimate of the abundance of this molecule.

\section{Discussion}

To investigate whether the data provide information on the formation of complex molecules, it is necessary to know what the possible formation paths are. We therefore report the formation reactions found in the literature for the detected molecules in Appendix A, and we bring up some key points regarding these formation routes in Sect. 5.1. Since low-mass protostars were thought to have insufficient luminosity to develop a hot core-type region, we will then investigate the potential dependence of the complex molecules' abundances on the luminosity (Sect. 5.2). In Sect. 5.3, we will analyze the hot corino data in view of the information given in Appendix A and look at the differences with their massive counterparts and with Galactic center clouds in Sects. 5.4 and 5.5, respectively.

\subsection{Notes on the formation routes of complex organic molecules}

For all the complex molecules considered here, we can see from Appendix A that grain-surface formation is a possible alternative to the "classical" gas-phase formation. In this classical view, an estimate of the formation and destruction timescales for $\mathrm{CH}_{3} \mathrm{OCH}_{3}$ indicates that destruction of this molecule is likely compensated by formation mechanisms (Sect. A.5). Regarding grain-surfaces processes, it is usually implicitly assumed that they are taking place during the cold phase preceding the warmup of the dust by the newly born star. However, as the protostar heats up, it is improbable that the dust temperature suddenly jumps from $\sim 10$ to $\sim 100 \mathrm{~K}$. Instead, it is more likely that the temperature "slowly" rises across the inner envelope, leading to a gradual heating of the grains (e.g., Viti et al. 2004; Garrod \& Herbst 2006). The effect of this gradual temperature change on grain-surface and gas-phase chemistry can be quite substantial. Indeed, the modeling by Garrod \& Herbst (2006) shows that gasphase and grain-surface chemistries are strongly coupled during the warm-up phase: molecules formed on the grain can evaporate and affect the gas-phase chemistry, whose products can reaccrete and in turn change the grain-surface chemistry. 
Table 5. Abundances of parent and daughter molecules in our sample of four low-mass protostars.

\begin{tabular}{lccccc}
\hline \hline Molecule & IRAS 16293 & IRAS4A & IRAS4B & IRAS2A & Ref. \\
\hline $\mathrm{H}_{2} \mathrm{CO}$ & $1 \times 10^{-7}$ & $2 \times 10^{-8}$ & $3 \times 10^{-6}$ & $2 \times 10^{-7}$ & $1,2,3$ \\
$\mathrm{CH}_{3} \mathrm{OH}$ & $1 \times 10^{-7}$ & $<1 \times 10^{-8}$ & $7 \times 10^{-7}$ & $3 \times 10^{-7}$ & 4 \\
$\mathrm{HCOOCH}_{3}-\mathrm{A}^{a}$ & $1.7 \times 10^{-7}$ & $3.4 \times 10^{-8}$ & $1.1 \times 10^{-6}$ & $<6.7 \times 10^{-7}$ & $5,2,6$ \\
$\mathrm{HCOOH}^{-8}$ & $6.2 \times 10^{-8}$ & $4.6 \times 10^{-9}$ & $<1.0 \times 10^{-6}$ & $<1.2 \times 10^{-7}$ & $5,2,6$ \\
$\mathrm{CH}_{3} \mathrm{OCH}$ & $2.4 \times 10^{-7}$ & $<2.8 \times 10^{-8}$ & $<1.2 \times 10^{-6}$ & $3.0 \times 10^{-8}$ & $5,2,6,7$ \\
$\mathrm{CH}_{3} \mathrm{CN}$ & $1.0 \times 10^{-8}$ & $1.6 \times 10^{-9}$ & $9.5 \times 10^{-8}$ & $8.7 \times 10^{-9}$ & $5,2,6$ \\
$\mathrm{C}_{2} \mathrm{H}_{5} \mathrm{CN}$ & $1.2 \times 10^{-8}$ & $<1.2 \times 10^{-9}$ & $<7.5 \times 10^{-7}$ & $<1.0 \times 10^{-7}$ & $5,2,6$ \\
\hline
\end{tabular}

Note - Except for $\mathrm{H}_{2} \mathrm{CO}$ and $\mathrm{CH}_{3} \mathrm{OH}$, for which Maret et al. $(2004,2005)$ took into account the effects of opacity to derive the abundances of these species, it was not possible to determine the optical thickness of the complex molecules' transitions, so that all the complex molecules' abundances should be considered as lower limits.

${ }^{a}$ We report here the abundances of the A form only of $\mathrm{HCOOCH}_{3}$, for which we have the highest number of rotational diagrams. Note that the abundance of the E form is usually very close to that of the A form (from IRAS 16293 and IRAS4A; Cazaux et al. 2003; Bottinelli et al. 2004a), so that the total $\mathrm{HCOOCH}_{3}$ abundance would be twice that of the A form.

References - (1) Ceccarelli et al. (2000c). (2) Bottinelli et al. (2004a). (3) Maret et al. (2004). (4) Maret et al. (2005). (5) Cazaux et al. (2003). (6) This work. (7) Jørgensen et al. (2005a).

Note that for some molecules, grain-surface formation is not only an alternative, but also apparently the only choice. This is the case for $\mathrm{HCOOCH}_{3}$ and even more so for $\mathrm{HCOOH}$ since it has been detected in the ices of star forming regions (e.g., Schutte et al. 1999; Keane et al. 2001), as well as of quiescent molecular clouds (Knez et al. 2005). In particular, the detection of icy $\mathrm{HCOOH}$ in quiescent clouds would indicate not only that $\mathrm{HCOOH}$ formation occurs on the grain surface, but also that it would pre-date the first phase of the star formation mechanism, supporting the theory of formation in the cold, rather than warmup phase. Whether the other complex organic molecules could also follow the same pattern cannot be commented upon since, to our knowledge, no other complex molecule has been detected in ices. This is because, unfortunately, the infrared spectra of complex organic molecules are not well known in this medium. The difficulty in determining the presence of these molecules lies in the fact that they may produce only slight shifts and broadenings, and that the peak positions are characteristic of functional groups, not molecular species (Tielens, priv. comm.). Hence, specific identifications are always somewhat ambiguous. Overall, it also stands out from Appendix A that more experiments on thermal surface chemistry at low temperatures are needed to check the feasibility of the outlined reactions. Cosmic ray processing of icy grain mantles may also be able to produce complex molecules, but this process requires more quantitative modeling to compare with observations.

\subsection{Luminosity dependence}

As mentioned previously, before the discovery of a hot corino around IRAS 16293, it was believed impossible to have chemically rich regions driven by gas-phase reactions following mantle evaporation around low-mass protostars. The argument was that, given the low luminosity of these objects, the regions where the ices sublimate would be so small that the gas crossing-time would be shorter than the time needed to form complex organic molecules in the gas phase (e.g., Schöier et al. 2002). This is in fact not the case since several hot corinos have now been discovered (Cazaux et al. 2003; Bottinelli et al. 2004a; Jørgensen et al. 2005a; this work). However, the question remains regarding the impact of the luminosity on the abundances of complex organic molecules in hot corinos.

Table 5 lists the measured abundances and upper limits for the four hot corinos. To remove the uncertainty on the sizes of the hot corinos, we choose to look at abundance ratios, in particular with respect to formaldehyde and methanol since these molecules have been proposed to be the parent molecules for complex oxygen-bearing species, if they are formed in the gasphase. These abundance ratios are plotted in Fig. 3 as a function of the bolometric luminosity of the low-mass protostars. For information, the abundance ratio for $\mathrm{CH}_{3} \mathrm{CN}$ is also plotted $\left(\mathrm{C}_{2} \mathrm{H}_{5} \mathrm{CN}\right.$ was not included due to the number of upper limits), although formaldehyde and methanol are not thought to be the parent molecules of nitrogen-bearing species (see Sect. A.4). Note that the abundance ratios of $\mathrm{CH}_{3} \mathrm{OCH}_{3}$ with respect to both $\mathrm{H}_{2} \mathrm{CO}$ and $\mathrm{CH}_{3} \mathrm{OH}$ in IRAS2A seem to be "outliers" compared to the other protostars and to other O-bearing molecules. However, recall that the $\mathrm{CH}_{3} \mathrm{OCH}_{3}$ abundance was taken from Jørgensen et al. (2005a) where it was derived from only one detected transition. Apart from the $\mathrm{CH}_{3} \mathrm{OCH}_{3}$ points and taking into account the uncertainties pertaining to abundance determination, we can see from Fig. 3 that the abundance ratios of complex molecules with respect to $\mathrm{H}_{2} \mathrm{CO}$ or $\mathrm{CH}_{3} \mathrm{OH}$ do not depend on the luminosity, in the range $\sim 5-30 L_{\odot}$. Since the abundances of $\mathrm{H}_{2} \mathrm{CO}$ or $\mathrm{CH}_{3} \mathrm{OH}$ are not themselves a function of luminosity (see Maret et al. 2004, 2005), then the absolute abundances of the complex species do not depend on the luminosity of the protostar. Whatever the formation mechanism, either in the gas phase or on the grain surfaces, the efficiency in forming complex organic molecules is largely constant in the range of studied luminosities. Since the luminosity, together with the density, defines the radius at which ices sublimate, this also implies that this efficiency is rather constant in the inner 200 AUs or so of the studied sources, despite the different involved densities (from $10^{6}$ to $10^{9} \mathrm{~cm}^{-3}$, Maret et al. 2004).

We also investigated the possible dependence of the abundance ratios on the ratio of submillimeter to bolometric luminosity, $L_{\mathrm{smm}} / L_{\mathrm{bol}}$, since it has been suggested as an indicator of evolutionary stage. We find that the abundance ratios do not depend on this parameter either. Maret et al. (2004) found an apparent anti-correlation between the inner abundance of $\mathrm{H}_{2} \mathrm{CO}$ and $L_{\mathrm{smm}} / L_{\text {bol }}$ and proposed that this could be explained if $L_{\mathrm{smm}} / L_{\mathrm{bol}}$ depends on the initial conditions of the protostars rather than their evolutionary stage. Indeed, more atomic hydrogen is available in less dense (i.e., with a higher $L_{\mathrm{smm}} / L_{\mathrm{bol}}$ ) environments, which leads to the formation of more $\mathrm{H}_{2} \mathrm{CO}$ and $\mathrm{CH}_{3} \mathrm{OH}$. If we plot the inner $\mathrm{CH}_{3} \mathrm{OH}$ abundance as a function of $L_{\mathrm{smm}} / L_{\mathrm{bol}}$, we also notice an apparent anti-correlation. Since the abundance ratios are roughly constant with different $L_{\mathrm{smm}} / L_{\text {bol }}$, then the absolute abundances of complex molecules 


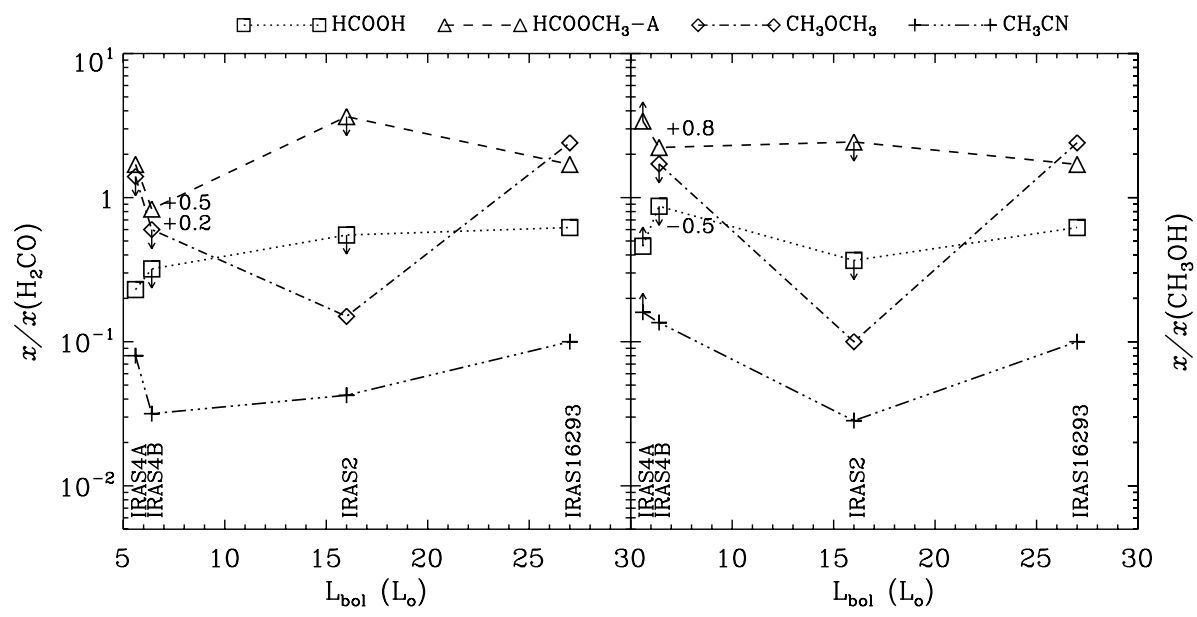

Fig. 3. The abundances of the observed species normalized to the $\mathrm{H}_{2} \mathrm{CO}$ (left) and $\mathrm{CH}_{3} \mathrm{OH}$ (right) abundances, plotted as a function of bolometric luminosity. Square, triangles, diamonds, and plus signs represent $\mathrm{HCOOH}$, $\mathrm{HCOOCH}_{3}, \mathrm{CH}_{3} \mathrm{OCH}_{3}$, and $\mathrm{CH}_{3} \mathrm{CN}$, respectively. The abundance for $\mathrm{CH}_{3} \mathrm{OCH}_{3}$ in IRAS2A was taken from Jørgensen et al. (2005a) and is likely underestimated (see text). Note that we only have an upper limit on the $\mathrm{CH}_{3} \mathrm{OH}$ abundance in the hot corino of IRAS4A, therefore, we did not plot the point corresponding to $\mathrm{CH}_{3} \mathrm{OCH}_{3}$ for which only an upper limit is available in that source. Also, we do not show $\mathrm{C}_{2} \mathrm{H}_{5} \mathrm{CN}$ since only upper limits are available in three of the four sources. should also be anti-correlated with this parameter. Following a similar line of thought as Maret et al. (2004) and assuming that complex O-bearing molecules form on grain surfaces via $\mathrm{H}, \mathrm{O}$, $\mathrm{OH}$, and/or $\mathrm{CH}_{3}$ additions, this anti-correlation could be indicative of these species being more readily available in less dense environments.

\subsection{Dependence on methanol and formaldehyde hot corino abundances}

Whether we consider gas-phase or grain-surface formation, $\mathrm{CH}_{3} \mathrm{OH}$ and $\mathrm{H}_{2} \mathrm{CO}$ appear as key molecules: in the first case, they have been suggested as parent molecules, in the second, they are known mantle constituents. It is therefore interesting to investigate the abundance ratios of complex molecules to $\mathrm{CH}_{3} \mathrm{OH}$ and $\mathrm{H}_{2} \mathrm{CO}$ as a function of $\mathrm{CH}_{3} \mathrm{OH}$ and $\mathrm{H}_{2} \mathrm{CO}$ abundances themselves. We plot these quantities for hot corinos in Figs. 4 and 5, respectively. From these figures, we can see that:

(i) The complex molecules in hot corinos have comparable abundance ratios, apparently independent of the $\mathrm{CH}_{3} \mathrm{OH}$ and $\mathrm{H}_{2} \mathrm{CO}$ abundances.

(ii) These abundance ratios are close to unity.

The implications are:

- In the case of gas-phase formation from methanol or formaldehyde: (i) and (ii) mean that, in all the hot corinos, the formation of complex molecules uses up a significant fraction of the parent molecules sublimated from the mantles. However, gas-phase chemistry does not seem able to reproduce this behavior. For example, the collapsing envelope model of Rodgers \& Charnley (2003; see also Rodgers \& Charnley 2001) predicts $\mathrm{CH}_{3} \mathrm{OCH}_{3}$ to $\mathrm{CH}_{3} \mathrm{OH}$ abundance ratios of only $10^{-2}-10^{-1}$. Similarly, Rodgers \& Charnley (2001) predicted $\mathrm{HCOOCH}_{3}$ to $\mathrm{CH}_{3} \mathrm{OH}$ abundance ratios $<7 \times 10^{-3}$, and we now know that they used too high a formation rate coefficient for $\mathrm{HCOOCH}_{3}$, so that the actual prediction should be even smaller (Horn et al. 2004). Therefore, either gas-phase models are not adequate, or complex molecules are not formed in the gas phase.

- In the case of grain-surface formation: (i) and (ii) show that complex molecules are as important mantle constituent as $\mathrm{CH}_{3} \mathrm{OH}$ and $\mathrm{H}_{2} \mathrm{CO}$. Observations of solid $\mathrm{HCOOH}$ and $\mathrm{CH}_{3} \mathrm{OH}$ along quiescent lines of sight by Knez et al. (2005) and in protostars (Keane et al. 2001) support this idea since the quoted $\mathrm{HCOOH}$ and $\mathrm{CH}_{3} \mathrm{OH}$ abundances yield abundance ratios of order unity, as we find for the hot corinos.
No observations of other complex molecules in the ices are available, but their presence cannot be excluded considering, as mentioned in Sect. 5.1, the difficulty of identifying their signature in infrared spectra.

Note that gas-phase reactions proposed in Sect. A.1 for the formation of $\mathrm{HCOOH}$ do not involve $\mathrm{CH}_{3} \mathrm{OH}$ or $\mathrm{H}_{2} \mathrm{CO}$. Therefore the conclusions mentioned for gas-phase formation do not apply to this molecule, whereas the analysis regarding grain-surface formation is still valid.

Using the methanol abundances derived by Jørgensen et al. (2005b) does not change the shape of Fig. 4 since their values are comparable to the ones derived by Maret et al. (2005). However, if we take formaldehyde abundances from Jørgensen et al. (2005b), we notice that the abundance ratios are larger than those plotted in Fig. 5 (as expected since Jørgensen et al. 2005b do not model any $\mathrm{H}_{2} \mathrm{CO}$ abundance jump), and that the abundance ratios are scattered by up to two orders of magnitude.

Overall, in the Maret et al. $(2004,2005)$ framework, our data are consistent with either gas-phase or grain-surface formation of complex molecules in hot corinos, with nonetheless more support for the later route. However, other scenarios could be considered. For example, complex molecules could form in the ISM, deplete onto grain mantles during the accretion phase and desorb as the protostar heats up its environment. If this formation in the ISM were true, we should be able to observe their low-energy transitions in dark clouds or in the cold envelopes of the protostars. For example, Remijan \& Hollis (2006) observed a transition of $\mathrm{HCOOH}$ with $E_{u}=3.2 \mathrm{~K}$ around IRAS $16293 \mathrm{~B}$ for which the spatial distribution is around (and not peaking at) the position determined from the continuum. We cannot therefore rule out this theory, but more observations are needed to support it.

\subsection{Comparison hot corinos-hot cores}

Due to the physical differences between hot corinos and hot cores, comparing these two types of objects can potentially tell us a lot about the parameters influencing the formation and evolution of complex molecules. We therefore searched the literature for the relevant abundances in hot cores to calculate abundance ratios in these objects as well. To our knowledge, there are only four hot cores (Sgr B2(N), Orion hot core, Orion compact ridge, and $\mathrm{W} 3\left(\mathrm{H}_{2} \mathrm{O}\right)$ ) for which data on all five O-bearing molecules studied here $\left(\mathrm{H}_{2} \mathrm{CO}, \mathrm{CH}_{3} \mathrm{OH}, \mathrm{HCOOH}\right.$, $\mathrm{HCOOCH}_{3}$, and $\mathrm{CH}_{3} \mathrm{OCH}_{3}$ ) are available, but we can find another seven sources (Sgr B2(M), G327.30-0.60, G34.26+0.15, 


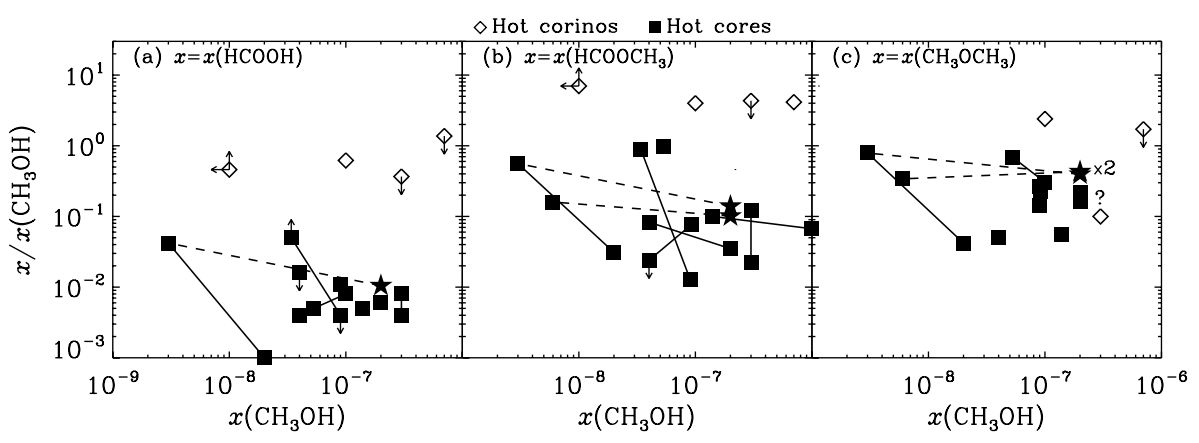

Fig. 4. Abundance ratios of complex O-bearing molecules to methanol, plotted as a function of the methanol abundance. Open diamonds represent hot corinos. Filled squares represent abundances ratios of hot cores, derived from beam-averaged column density analysis. Squares linked by a solid line represent abundance determinations from different authors (Gibb et al. 2000a and references therein, Ikeda et al. 2001). Stars represent the hot cores of SgrB2 (N) and (M), where an analysis of the methanol emission similar to what done in the hot corinos has been carried out (Nummelin et al. 2000). The dotted lines connect the SgrB2 (N) and (M) hot cores (stars) to the squares corresponding to the cold envelopes of these sources. See the text for further details. Note that panel (b) represents the total $\mathrm{HCOOCH}_{3}$ abundances, that is twice the abundances of the A form quoted in Table 5. The point corresponding to $\mathrm{CH}_{3} \mathrm{OCH}_{3}$ in IRAS4A is not represented due to both $\mathrm{CH}_{3} \mathrm{OCH}_{3}$ and $\mathrm{CH}_{3} \mathrm{OH}_{\text {abundances being }}$ upper limits in this source. The question mark refers to the ratio in IRAS2A and indicates that the $\mathrm{CH}_{3} \mathrm{OCH}_{3}$ abundance is likely underestimated in this source (see Sect. 5.2).

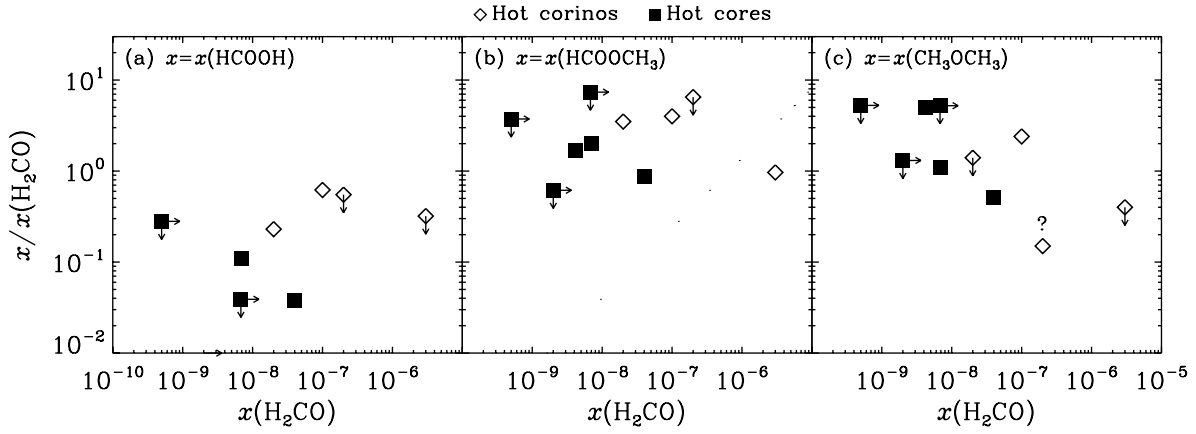

Fig. 5. Abundance ratios of complex O-bearing molecules to formaldehyde, plotted as a function of the formaldehyde abundance. Open diamonds represent hot corinos. Filled squares represent abundances ratios of hot cores, derived by beam-averaged column density analysis.
NGC 6334(I), W51 e1/e2, G31.41+0.31, and G10.47+0.03) for which $\mathrm{CH}_{3} \mathrm{OH}$ and at least two of the complex molecules molecules $\left(\mathrm{HCOOH}, \mathrm{HCOOCH}_{3}\right.$, and $\mathrm{CH}_{3} \mathrm{OCH}_{3}$ ) have measured abundances. Data are taken from Gibb et al. (2000a) and references therein, and from Ikeda et al. (2001).

Figures 4 and 5 plot the abundance ratios of massive hot cores, as derived from the literature. The first thing to notice is that the abundance ratios in the massive hot cores have been derived by beam-averaged column densities, except for SgrB2 (N) and (M), where Nummelin et al. (2000) have carried out an analysis of the methanol emission similar to what has been done for the hot corinos, namely disentangling the hot core from the cold outer envelope contribution in the beam. For a given complex molecule, if a source was listed in both Gibb et al. (2000a) and Ikeda et al. (2001), then two abundance ratios were derived from the different authors. In this case, the two values are reported in Fig. 4 and linked with a solid line. Disturbingly, they can differ by almost two orders of magnitude, and the cause of this discrepancy is unclear. In Fig. 4, we also report the results obtained from the hot core + outer envelope analysis of $\mathrm{CH}_{3} \mathrm{OH}$ emission in SgrB2 (N) and (M), to show the uncertainty associated with the different methods of abundance ratios' determinations. As can be seen, there is only a factor 4 difference in the abundance ratios if the hot core or the outer envelope abundance of methanol is used.

In principle, the different methods used to derive the abundance ratios in hot cores (beam-averaged column density) and corinos (full density and chemical structure analysis) may lead to such a different result that the comparison may be meaningless. However, while this would certainly be the case for the absolute values of the abundances, the abundance ratios suffer much less from the different methods, as shown by the "small" factor 4 in the SgrB2 (N) and (M) sources (Nummelin et al. 2000). In practice, the beam-averaged abundance ratios are very different from the reality only if the spatial distributions of the different molecules in the considered source are different, and/or if there is a large contribution from the outer envelope with respect to the hot core. While a direct measure of the molecular emission extent would require carrying out interferometric observations, the available data can give a good hint on where the emission originates from, by looking at the rotational temperature, $T_{\text {rot }}$. As a matter of fact, the $T_{\text {rot }}$ of the hot cores considered in this study (with the possible exception of the $\mathrm{H}_{2} \mathrm{CO}$ and $\mathrm{HCOOH}$ ) are implying that the emission is indeed dominated by the hot cores rather than the cold envelope (which may also be due to an observational bias, that is, if the observed transitions are probing warm rather than cold gas). In this respect, therefore, we think that the comparison between hot corinos and hot cores shown in Fig. 4 is reliable. There is, however, more uncertainty associated with the hot cores' abundance ratios reported in Fig. 5, since $\mathrm{H}_{2} \mathrm{CO}$ emission could have an important contribution from the cold envelope.

The abundance ratios with respect to $\mathrm{CH}_{3} \mathrm{OH}$ and $\mathrm{H}_{2} \mathrm{CO}$ were noticed to be roughly constant for hot corinos. Regarding hot cores, the ratios seem to decrease with increasing $\mathrm{CH}_{3} \mathrm{OH}$ or $\mathrm{H}_{2} \mathrm{CO}$ abundance, but the data are also consistent with a constant ratio with a larger scatter. In any case, it is clear from these figures that the abundance ratios with respect to methanol in hot cores are lower than in hot corinos by 1-2 orders of magnitude, whereas abundance ratios with respect to formaldehyde 
are comparable in hot corinos and hot cores. We also notice that the $\mathrm{HCOOH}$ abundance ratios in hot cores are about one order of magnitude lower than the $\mathrm{HCOOCH}_{3}$ and $\mathrm{CH}_{3} \mathrm{OCH}_{3}$ abundance ratios, whereas they are lower by only about a factor four in hot corinos. For completeness, we considered how Figs. 4 and 5 would change if we were to use beam-averaged abundances ratios in hot corinos, as in hot cores. In this case, abundance ratios in hot corinos would be smaller by a factor $\lesssim 10$ : ratios for $\mathrm{HCOOCH}_{3}$ and $\mathrm{CH}_{3} \mathrm{OCH}_{3}$ would become comparable in the two types of objects, while $\mathrm{HCOOH}$ ratios would still be larger in hot corinos than in hot cores. This is very likely due to the relative larger contribution of methanol and formaldehyde emission in the cold envelope in low mass with respect to high mass protostars. Indeed, high energy transitions are more easily detected in high than low mass protostars (e.g., Comito et al. 2005; Schilke et al. 2001, 1997; Blake et al. 1995), which support the above interpretation.

Whether considering beam-averaged ratios in all objects or not, Fig. 4 (and to a lesser extent Fig. 5) show that hot corinos are not just scaled versions of hot cores and that in fact, complex molecules are relatively more abundant in hot corinos than in hot cores. This conclusion would still be valid if we assume $\mathrm{CH}_{3} \mathrm{OH}$ and $\mathrm{H}_{2} \mathrm{CO}$ abundances from Jørgensen et al. (2005b) since, as mentioned in the previous section, the abundance ratios with respect to $\mathrm{CH}_{3} \mathrm{OH}$ and $\mathrm{H}_{2} \mathrm{CO}$ would be similar and higher, respectively, so in any case, they would be higher than the abundance ratios in massive hot cores. A possible explanation for the difference between hot cores and hot corinos could lie in the grain mantle composition. Indeed, we already know that the levels of deuteration differ in these two types of objects, more specifically that extreme deuteration occurs in low-mass but not in high-mass protostars (e.g., Ceccarelli et al. 2006, and references therein). Moreover, Boogert et al. (2004) summarize the abundances of some mantle constituents $\left(\mathrm{H}_{2} \mathrm{O}, \mathrm{CO}, \mathrm{CO}_{2}, \mathrm{CH}_{3} \mathrm{OH}, \mathrm{OCN}^{-}\right)$in two low-mass and high-mass embedded protostars, and large differences can also be seen there. It would therefore not be surprising that this would also be the case for complex molecules if they formed on the grain surfaces. The reason could be, for example, that hot corinos are preceded by a longer cold phase during which grain-surface reactions are at play, so that complex molecules in hot cores would not have time to become an important grain-mantle constituent.

Alternatively, the difference in abundance ratios could be due to a difference in the chemistry, since this depends on environmental parameters such as the density and temperature of the gas. For example, the model of Rodgers \& Charnley (2001) shows that the predicted abundances are different at 100 or $300 \mathrm{~K}$. Also, considering for example the methyl formate, the gas-grain model in the warm-up phase presented in Garrod \& Herbst (2006) shows that the gas-phase formation of this molecule is more efficient at low temperatures, and that the grain-surface pathway leads to higher abundances the longer the dust temperature remains in the $20-40 \mathrm{~K}$ range. Either way, formation of complex molecules during the warm-up stage of the protostar would be more efficient in low- than in high-mass environments. However, observations performed towards Galactic center clouds (see Sect. 5.5) are acting against this theory since complex molecules are observed in these clouds although they are not subject to a warm-up phase.

Finally, recall that all the proposed grain-surface formation routes of complex molecules involved UV or cosmic-ray processing. Hot cores/corinos are shielded from external UV radiation fields, but low-mass protostars, unlike massive ones, are known to be powerful X-ray sources (e.g.,
Feigelson \& Montmerle 1999). X-rays have already been proposed to be the reason for the presence of calcite in the lowmass protostars (Chiavassa et al. 2005; Ceccarelli et al. 2002b). In our case, X-rays emitted by Class 0 objects could provide the necessary energy to produce a large number of radicals and hence a large amount of complex molecules on the grain surfaces, thereby explaining the larger abundance ratios observed in hot corinos compared to hot cores.

\subsection{Comparison with Galactic center clouds}

Hot cores/corinos are not the only objects where complex molecules have been observed. Indeed, Requena-Torres et al. (2006) have carried out a survey of complex O-bearing molecules in Galactic center (GC) clouds. These clouds are known to possess a warm $(>100 \mathrm{~K})$ and not too dense $\left(\sim 10^{4} \mathrm{~cm}^{-3}\right)$ gas (Hüttemeister et al. 1993; Rodríguez-Fernández et al. 2000; Ceccarelli et al. 2002a). This warm component is probably caused by shocks (e.g., Flower et al. 1995; Rodríguez-Fernández et al. 2004) arising from cloud-cloud collisions (Hüttemeister et al. 1993, 1998). These shocks are thought to be at the origin of the enhanced $\mathrm{NH}_{3}$ (Flower et al. 1995), $\mathrm{SiO}$ (Martín-Pintado et al. 1997), and $\mathrm{C}_{2} \mathrm{H}_{5} \mathrm{OH}$ (Martín-Pintado et al. 2001) abundances.

Surprisingly enough, considering the very different physical environments between GC clouds and hot corinos, Requena-Torres et al. (2006) found, as we do in the present work for hot corinos, that the ratios of the abundances with respect to $\mathrm{CH}_{3} \mathrm{OH}$ (which are comparable to those reported in Fig. 4 for hot cores) are approximately constant and do not depend on the $\mathrm{CH}_{3} \mathrm{OH}$ abundance. The authors conclude that the gasphase abundances of the organic molecules they observed in GC clouds are likely due to the formation of these molecules on the grain surfaces and their release in the gas-phase from sputtering/erosion of the grain mantles by the shocks, as it is the case for ammonia, silicon oxide, and ethanol.

Note that there is evidence for the presence of X-rays in these GC clouds (Martín-Pintado et al. 2000). These authors suggest that X-rays could contribute to the formation of molecules on grain surfaces and evaporate small dust grains. This theory adds some support to the possible role played by X-rays in the formation of complex organic molecules.

\section{Conclusion}

In this paper, we presented the detections of methyl formate and/or methyl cyanide in the low-mass protostars IRAS4B and IRAS2A, confirming the presence of a hot corino in their inner envelope. The conclusions arising from the analysis of these observations combined with data on the two other hot corinos (IRAS 16293 and IRAS4A) are:

- Hot corinos are a common phase in the formation of solartype protostars and complex organic molecules are ubiquitous in Class 0 protostars.

- The absolute abundances (i.e., with respect to $\mathrm{H}_{2}$ ) of complex molecules in hot corinos do not depend on the bolometric luminosity.

- Abundance ratios of complex molecules' abundances to $\mathrm{CH}_{3} \mathrm{OH}$ or $\mathrm{H}_{2} \mathrm{CO}$ abundances $\left(x / x\left(\mathrm{CH}_{3} \mathrm{OH}\right)\right.$ and $x / x\left(\mathrm{H}_{2} \mathrm{CO}\right)$ are of order unity and do not depend on $\mathrm{CH}_{3} \mathrm{OH}$ or $\mathrm{H}_{2} \mathrm{CO}$ abundances, indicating that complex molecules form on grain surfaces or that gas-phase models have to be revised. 
Furthermore, we compared hot corinos with massive hot cores. Keeping in mind the different methods of abundance determination used in the two types of objects, we found that $x / x\left(\mathrm{CH}_{3} \mathrm{OH}\right)$ and $x / x\left(\mathrm{H}_{2} \mathrm{CO}\right)$ in hot cores are relatively lower than in hot corinos, showing that complex molecules are relatively more important in hot corinos, a difference that can be explained in the case of grain-surface synthesis.

Overall, although there is no absolute proof, there is circumstantial evidence for the formation of complex organic molecules on grain surfaces, either in the cold phase preceding the begin of the star formation process, or in the warm-up phase following the birth of the protostar. In this scenario, there is also a possibility that X-rays emitted by low-mass protostars participate in the formation of these complex molecules. Finally, it clearly stands out from this work that not only more data (single-dish, interferometric, and high-energy transitions) are needed, but also that laboratory studies of grain-surface reactions are necessary to answer of the question of which, why, where, and how complex molecules are formed.

Acknowledgements. We wish to thank Miguel Requena-Torres and his coauthors, as well as Robin Garrod and Eric Herbst for communicating the results of their work prior to publication (at the time of submission). We are also very grateful to Malcom Walmsley and the anonymous referee for their careful reading of the manuscript and for suggestions that helped to improve the paper.

\section{References}

Allen, M., \& Robinson, G. W. 1977, ApJ, 212, 396

André, P., Ward-Thompson, D., \& Barsony, M. 2000, Protostars and Planets IV, 59

Blake, G. A., Sandell, G., van Dishoeck, E. F., et al. 1995, ApJ, 441, 689

Blake, G. A., Sutton, E. C., Masson, C. R., \& Phillips, T. G. 1987, ApJ, 315, 621

Boogert, A. C. A., Pontoppidan, K. M., Lahuis, F., et al. 2004, ApJS, 154, 359

Bottinelli, S., Ceccarelli, C., Lefloch, B., et al. 2004a, ApJ, 615, 354

Bottinelli, S., Ceccarelli, C., Neri, R., et al. 2004b, ApJ, 617, L69

Bottinelli, S., Ceccarelli, C., Lefloch, B., \& Williams, J. P. 2006, A\&A, submitted

Caselli, P., Hasegawa, T. I., \& Herbst, E. 1993, ApJ, 408, 548

Cazaux, S., Tielens, A. G. G. M., Ceccarelli, C., et al. 2003, ApJ, 593, L51

Ceccarelli, C. 2004, in Star Formation in the Interstellar Medium: In Honor of David Hollenbach, ASP Conf. Ser., 323, 195

Ceccarelli, C., Hollenbach, D. J., \& Tielens, A. G. G. M. 1996, ApJ, 471, 400

Ceccarelli, C., Castets, A., Caux, E., et al. 2000a, A\&A, 355, 1129

Ceccarelli, C., Loinard, L., Castets, A., Faure, A., \& Lefloch, B. 2000b, A\&A, 362,1122

Ceccarelli, C., Loinard, L., Castets, A., Tielens, A. G. G. M., \& Caux, E. 2000c, A\&A, 357, L9

Ceccarelli, C., Baluteau, J.-P., Walmsley, M., et al. 2002a, A\&A, 383, 603

Ceccarelli, C., Caux, E., Tielens, A. G. G. M., et al. 2002b, A\&A, 395, L29

Ceccarelli, C., Caselli, P., Herbst, E., Tielens, A. G. M. M., \& Caux, E. 2006, in Protostars and Planets V

Černis, K. 1990, Ap\&SS, 166, 315

Chandler, C. J., Brogan, C. L., Shirley, Y. L., \& Loinard, L. 2005, ApJ, 632, 371

Charnley, S. B., \& Rodgers, S. D. 2005, in IAU Symp., 262

Charnley, S. B., Tielens, A. G. G. M., \& Millar, T. J. 1992, ApJ, 399, L71

Charnley, S. B., Tielens, A. G. G. M., \& Rodgers, S. D. 1997, ApJ, 482, L203

Chiavassa, A., Ceccarelli, C., Tielens, A. G. G. M., Caux, E., \& Maret, S. 2005, A\&A, 432, 547

Combes, F., Q-Rieu, N., \& Wlodarczak, G. 1996, A\&A, 308, 618

Comito, C., Schilke, P., Phillips, T. G., et al. 2005, ApJS, 156, 127

Ehrenfreund, P., \& Charnley, S. B. 2000, ARA\&A, 38, 427

Feigelson, E. D., \& Montmerle, T. 1999, ARA\&A, 37, 363

Flower, D. R., Pineau des Forets, G., \& Walmsley, C. M. 1995, A\&A, 294, 815

Garrod, R. T., \& Herbst, E. 2006, A\&A, submitted

Gibb, E., Nummelin, A., Irvine, W. M., Whittet, D. C. B., \& Bergman, P. 2000a, ApJ, 545, 309
Gibb, E. L., Whittet, D. C. B., Schutte, W. A., et al. 2000b, ApJ, 536, 347 Hasegawa, T. I., \& Herbst, E. 1993, MNRAS, 261, 83

Herbst, E. 2005, J. Phys. Chem. A, 109, 4017

Horn, A., Møllendal, H., Sekiguchi, O., et al. 2004, ApJ, 611, 605

Hudson, R. L., \& Moore, M. H. 1999, Icarus, 140, 451

Hüttemeister, S., Wilson, T. L., Bania, T. M., \& Martín-Pintado, J. 1993, A\&A, 280,255

Hüttemeister, S., Dahmen, G., Mauersberger, R., et al. 1998, A\&A, 334, 646

Ikeda, M., Ohishi, M., Nummelin, A., et al. 2001, ApJ, 560, 792

Irvine, W. M., Friberg, P., Kaifu, N., et al. 1990, A\&A, 229, L9

Jørgensen, J. K., Schöier, F. L., \& van Dishoeck, E. F. 2002, A\&A, 389, 908

Jørgensen, J. K., Bourke, T. L., Myers, P. C., et al. 2005a, ApJ, 632, 973

Jørgensen, J. K., Schöier, F. L., \& van Dishoeck, E. F. 2005b, A\&A, 437, 501

Keane, J. V., Tielens, A. G. G. M., Boogert, A. C. A., Schutte, W. A., \& Whittet,

D. C. B. 2001, A\&A, 376, 254

Knee, L. B. G., \& Sandell, G. 2000, A\&A, 361, 671

Knez, C., Boogert, A. C. A., Pontoppidan, K. M., et al. 2005, ApJ, 635, L145

Kuan, Y.-J., Huang, H.-C., Charnley, S. B., et al. 2004, ApJ, 616, L27

Lefloch, B., Castets, A., Cernicharo, J., \& Loinard, L. 1998, ApJ, 504, L109

Leung, C. M., Herbst, E., \& Huebner, W. F. 1984, ApJS, 56, 231

Liu, S.-Y., \& Snyder, L. E. 1999, ApJ, 523, 683

Liu, S.-Y., Mehringer, D. M., \& Snyder, L. E. 2001, ApJ, 552, 654

Looney, L. W., Mundy, L. G., \& Welch, W. J. 2000, ApJ, 529, 477

Lovas, F. J., \& Dragoset, R. A. 2004, NIST Recommended Rest Frequencies for

Observed Interstellar Molecular Microwave Transitions - 2002 Revision

Maret, S., Ceccarelli, C., Caux, E., et al. 2004, A\&A, 416, 577

Maret, S., Ceccarelli, C., Tielens, A. G. G. M., et al. 2005, A\&A, 442, 527

Martín-Pintado, J., de Vicente, P., Fuente, A., \& Planesas, P. 1997, ApJ, 482, L45

Martín-Pintado, J., de Vicente, P., Rodríguez-Fernández, N. J., Fuente, A., \& Planesas, P. 2000, A\&A, 356, L5

Martín-Pintado, J., Rizzo, J. R., de Vicente, P., Rodríguez-Fernández, N. J., \& Fuente, A. 2001, ApJ, 548, L65

Müller, H. S. P., Thorwirth, S., Roth, D. A., \& Winnewisser, G. 2001, A\&A, 370, L49

Müller, H. S. P., Schlöder, F., Stutzki, J., et al. 2005, in IAU Symp., 30

Nummelin, A., Bergman, P., Hjalmarson, A., et al. 2000, ApJS, 128, 213

Peeters, Z., Rodgers, S. D., Charnley, S. B., et al. 2006, A\&A, 445, 197

Pickett, H. M., Poynter, R. L., Cohen, E. A., et al. 1998, J. Quant. Spec. Radiat. Transf., 60, 883

Reipurth, B., Rodríguez, L. F., Anglada, G., \& Bally, J. 2002, AJ, 124, 1045

Remijan, A., \& Hollis, J. M. 2006, ApJ, in press

Requena-Torres, M. A., Martín-Pintado, J., Rodríguez-Franco, A., et al. 2006, A\&A, 455, 971

Rodgers, S. D., \& Charnley, S. B. 2001, ApJ, 546, 324

Rodgers, S. D., \& Charnley, S. B. 2003, ApJ, 585, 355

Rodríguez-Fernández, N. J., Martín-Pintado, J., de Vicente, P., et al. 2000, A\&A, 356,695

Rodríguez-Fernández, N. J., Martín-Pintado, J., Fuente, A., \& Wilson, T. L. 2004, A\&A, 427, 217

Rowe, B. R., Dupeyrat, G., Marquette, J. B., et al. 1984, J. Chem. Phys., 80, 241

Sakai, N., Sakai, T., \& Yamamoto, S. 2006, PASJ, 58, L15

Sandell, G., Knee, L. B. G., Aspin, C., Robson, I. E., \& Russell, A. P. G. 1994, A\&A, 285, L1

Schilke, P., Groesbeck, T. D., Blake, G. A., \& Phillips, T. G. 1997, ApJS, 108, 301

Schilke, P., Benford, D. J., Hunter, T. R., Lis, D. C., \& Phillips, T. G. 2001, ApJS, 132, 281

Schöier, F. L., Jørgensen, J. K., van Dishoeck, E. F., \& Blake, G. A. 2002, A\&A, 390, 1001

Schutte, W. A., Boogert, A. C. A., Tielens, A. G. G. M., et al. 1999, A\&A, 343, 966

Sorrell, W. H. 2001, ApJ, 555, L129

Stäuber, P., Doty, S. D., van Dishoeck, E. F., \& Benz, A. O. 2005, A\&A, 440, 949

Tielens, A. G. G. M., \& Hagen, W. 1982, A\&A, 114, 245

Viti, S., Collings, M. P., Dever, J. W., McCoustra, M. R. S., \& Williams, D. A. 2004, MNRAS, 354, 1141

Ward-Thompson, D., André, P., Crutcher, R., et al. 2006, in Protostars and Planets V

White, R. J., Greene, T. P., Doppmann, G. W., Covey, K. R., \& Hillenbrand, L. A. 2006, in Protostars and Planets V 
S. Bottinelli et al.: Hot corinos in NGC 1333-IRAS4B and IRAS2A, Online Material $p 1$

\section{Online Material}




\section{Appendix A: Theories of complex molecules formation}

In this appendix, we review the different reactions that have been proposed for the formation of complex species. These reactions can occur in the gas-phase or on grain surfaces. Gas-phase reactions usually involve $\mathrm{H}_{2} \mathrm{CO}$ or $\mathrm{CH}_{3} \mathrm{OH}$ as precursors, or parents. These are known to form on grain surfaces (e.g., Tielens \& Hagen 1982; Charnley et al. 1997) and evaporate in the gasphase where they undergo further reaction to form more complex, or daughter, molecules. Grain-surface reactions generally consist of $\mathrm{H}$ or $\mathrm{O}$ additions and radical-radical reactions.

\section{A.1. Formic acid}

Both gas-phase and grain-surface processes have been proposed for the formation of $\mathrm{HCOOH}$. In the gas-phase model of Leung et al. (1984), the precursor ion of $\mathrm{HCOOH}, \mathrm{HCOOH}_{2}^{+}$, is produced via the radiative association

$\mathrm{H}_{2} \mathrm{O}+\mathrm{HCO}^{+} \rightarrow \mathrm{HCOOH}_{2}^{+}+h v$

followed by dissociative electron recombination to produce $\mathrm{HCOOH}$. However, Irvine et al. (1990) indicate that they believe that this reaction does not occur due to a competitive exothermic reaction to form $\mathrm{H}_{3} \mathrm{O}^{+}$and $\mathrm{CO}$. Instead, they attribute the gas-phase formation of $\mathrm{HCOOH}$ to occur via an ion-molecule reaction followed again by a dissociative electron recombination

$$
\begin{aligned}
& \mathrm{CH}_{4}+\mathrm{O}_{2}^{+} \rightarrow \mathrm{HCOOH}_{2}^{+}+\mathrm{H} \\
& \mathrm{HCOOH}_{2}^{+}+\mathrm{e}^{-} \rightarrow \mathrm{HCOOH}+\mathrm{H} .
\end{aligned}
$$

Reaction (A.2) has been measured in the laboratory and found to be quite rapid at low temperature (Rowe et al. 1984). Moreover, the calculated $\mathrm{HCOOH}$ abundance agrees with the values observed by Irvine et al. (1990) in the dark cloud L134 N.

On grain surfaces, Tielens \& Hagen (1982) proposed the formation of $\mathrm{HCOOH}$ through successive additions of $\mathrm{H}, \mathrm{O}$, and $\mathrm{H}$ to solid-state $\mathrm{CO}$ :

$$
\mathrm{CO} \stackrel{+\mathrm{H}}{\longrightarrow} \mathrm{HCO} \stackrel{+\mathrm{O}}{\longrightarrow} \mathrm{HCOO} \stackrel{+\mathrm{H}}{\longrightarrow} \mathrm{HCOOH} .
$$

However, radiolysis experiments (simulating the processing of interstellar ices by cosmic rays) by Hudson \& Moore (1999) showed that $\mathrm{HCOOH}$ could form in $\mathrm{H}_{2} \mathrm{O}-\mathrm{CO}$ mixed ices via the following sequence:

$$
\begin{aligned}
& \mathrm{H}+\mathrm{CO} \rightarrow \mathrm{HCO} \\
& \mathrm{HCO}+\mathrm{OH} \rightarrow \mathrm{HCOOH} .
\end{aligned}
$$

The models of Hasegawa \& Herbst (1993) are able to reproduce the abundances observed in massive hot cores for ages larger than $10^{5}-10^{6} \mathrm{yr}$. Finally, the grain-surface formation of $\mathrm{HCOOH}$ is supported by the interferometric observations of Sgr B2 and W51 by Liu et al. (2001), and would also be consistent with observations of this molecule in the ices surrounding the massive protostar W33A (Schutte et al. 1999; Gibb et al. 2000b).

\section{A.2. Methyl formate}

The commonly accepted formation path for $\mathrm{HCOOCH}_{3}$ starts with the reaction between protonated methanol and formaldehyde to form protonated methyl formate and molecular hydrogen:

$\left[\mathrm{CH}_{3} \mathrm{OH}_{2}\right]^{+}+\mathrm{H}_{2} \mathrm{CO} \rightarrow\left[\mathrm{HC}(\mathrm{OH}) \mathrm{OCH}_{3}\right]^{+}+\mathrm{H}_{2}$ followed by dissociative recombination of $\left[\mathrm{HC}(\mathrm{OH}) \mathrm{OCH}_{3}\right]^{+}$ with electrons to form $\mathrm{HCOOCH}_{3}$ (Blake et al. 1987). However, laboratory and theoretical work by Horn et al. (2004) indicates the existence of a very large activation energy for reaction (A.7), so that the later cannot lead to the formation of protonated methyl formate. Therefore, the formation of methyl formate in hot cores cannot occur via this reaction. Horn et al. (2004) searched for more favorable transitions between the reactants and products of reaction (A.7), but were unsuccessful. These authors also investigated reactions involving other abundant species in hot cores, such as protonated formaldehyde and CO. They show that none of the studied processes produces enough methyl formate to explain the observed abundances. However, they also state that one possibility for producing more methyl formate is that formic acid would be synthesized on grain surfaces and desorbed into the gas phase, in which case the reaction:

$$
\mathrm{CH}_{3}^{+}+\mathrm{HCOOH} \rightarrow \mathrm{HC}(\mathrm{OH}) \mathrm{OCH}_{3}^{+}+h v
$$

would play a significant role.

The downfall is that Horn et al. (2004) find that even if $\mathrm{HCOOH}$ were injected with an abundance one order of magnitude higher than observed in OMC-1, their model still predicts a $\mathrm{HCOOCH}_{3}$ abundance between one and two orders of magnitude below the observed value in this source. Overall, in the light of their work, Horn et al. (2004) conclude that no gasphase route seem able to reproduce the observed abundances of $\mathrm{HCOOCH}_{3}$, and hence that this molecule should be produced, at least in part, on grain surfaces. As pointed out in Sect. A.1, there is additional evidence in favor of $\mathrm{HCOOH}$ being synthesized on grain surfaces.

Two schemes for grain-surface formation of $\mathrm{HCOOCH}_{3}$ have been proposed, but none of them has undergone laboratory investigation yet:

- Formation from precursors $\mathrm{CO}, \mathrm{O}, \mathrm{C}$, and $\mathrm{H}$ landing on grain (Herbst 2005):

$$
\begin{aligned}
& \mathrm{CO}+\mathrm{H} \rightarrow \mathrm{HCO} \\
& \mathrm{C} \stackrel{+\mathrm{H}}{\mathrm{CH}} \stackrel{+\mathrm{H}}{\longrightarrow} \mathrm{CH}_{2} \stackrel{+\mathrm{H}}{\longrightarrow} \mathrm{CH}_{3} \stackrel{+\mathrm{O}}{\longrightarrow} \mathrm{CH}_{3} \mathrm{O} \\
& \mathrm{CH}_{3} \mathrm{O}+\mathrm{HCO} \rightarrow \mathrm{HCOOCH}_{3} .
\end{aligned}
$$

Charnley \& Rodgers (2005) mention that many radicals (like $\mathrm{CH}_{3} \mathrm{O}$ and $\mathrm{HCO}$ ) could form in close proximity via the hot secondary electron generated by the passage of a cosmic ray through the ice. In this case, it would ensue that radicalradical reactions such as (A.11) could occur efficiently.

- Sorrell (2001) proposed a model in which the photoprocessing of grain mantles by UV starlight creates a high concentration of radicals in the bulk interior of mantles. Grain-grain collisions then provide excess heat causing radical-radical reactions to occur and form large organic molecules. In this scheme, $\mathrm{HCOOCH}_{3}$ would be produced from the reaction between the carboxyl acid $(\mathrm{COOH})$ and the methyl group $\left(\mathrm{CH}_{3}\right)$ in the following way:

$$
\begin{aligned}
& \mathrm{CO}+\mathrm{OH} \rightarrow \mathrm{COOH} \text { or } \\
& \quad \mathrm{HCO}+\mathrm{HCO} \rightarrow \mathrm{COOH}+\mathrm{CH} \\
& \mathrm{CH} \stackrel{+\mathrm{H}}{\longrightarrow} \mathrm{CH}_{2} \stackrel{+\mathrm{H}}{\longrightarrow} \mathrm{CH}_{3} \\
& \mathrm{COOH}+\mathrm{CH}_{3} \rightarrow \mathrm{HCOOCH}_{3} .
\end{aligned}
$$

However, any grain-surface chemistry preceding the hot core/corino phase would occur in a very dense and highly 
visually extinct environment, hence well shielded from UV starlight. Therefore, such a UV photolysis of grains is unlikely to happen, as pointed out by Peeters et al. (2006). Nevertheless, the radical-radical reaction (A.14) could still be a possible formation path for $\mathrm{HCOOCH}_{3}$ via cosmic ray processing.

\section{A.3. Dimethyl ether}

$\mathrm{CH}_{3} \mathrm{OCH}_{3}$ was proposed by Blake et al. (1987) to form in the gas-phase by methyl cation transfer to methanol, followed by electron dissociative recombination:

$$
\begin{aligned}
& \mathrm{CH}_{3} \mathrm{OH}_{2}^{+}+\mathrm{CH}_{3} \mathrm{OH} \rightarrow \mathrm{CH}_{3} \mathrm{OCH}_{4}^{+}+\mathrm{H}_{2} \mathrm{O} \\
& \mathrm{CH}_{3} \mathrm{OCH}_{4}^{+}+\mathrm{e}^{-} \rightarrow \mathrm{CH}_{3} \mathrm{OCH}_{3}+\mathrm{H} .
\end{aligned}
$$

Peeters et al. (2006) claim that their models support such a gasphase route if the methanol abundance is of the order $10^{-6}$ or more, as it is the case for the OMC-1 hot core (recall that for hot corinos, $\left.X\left(\mathrm{CH}_{3} \mathrm{OH}\right) \leq 3 \times 10^{-7}\right)$. However, Ceccarelli et al. (2006) note that experimental measurements of the neutral products of dissociative recombination reactions show that two-body products such as in reaction (A.16) are often minor channels. Therefore, gas-phase formation of $\mathrm{CH}_{3} \mathrm{OCH}_{3}$ is plausible but not demonstrated.

On the grains, $\mathrm{CH}_{3} \mathrm{OCH}_{3}$ could be produced by a similar scheme as for $\mathrm{HCOOCH}_{3}$, that is cosmic ray processing followed by the radical-radical reaction $\mathrm{CH}_{3}+\mathrm{CH}_{3} \mathrm{O} \rightarrow \mathrm{CH}_{3} \mathrm{OCH}_{3}$ (Allen \& Robinson 1977). As in the case of $\mathrm{HCOOCH}_{3}$, this reaction has not been validated by laboratory studies.

\section{A.4. Methyl and ethyl cyanide}

For completeness, we mention here the possible formation routes of these two molecules. However, we do not discuss them any further in this study, due to the lack of data on potential parents such as $\mathrm{NH}_{3}$. Two substantially different formation routes for $\mathrm{CH}_{3} \mathrm{CN}$ have been proposed in the literature: either in the gas-phase or on grain surfaces.

- In the Rodgers \& Charnley (2001) chemical model of massive hot cores, $\mathrm{CH}_{3} \mathrm{CN}$ is synthesized from $\mathrm{NH}_{3}$ in the following way: $\mathrm{HCN}$ is synthesized from the reaction between $\mathrm{NH}_{3}$ and $\mathrm{C}^{+}$(yielding $\mathrm{HCNH}^{+}$, reaction (A.17)), followed by electron recombination or proton transfer to ammonia (A.18). $\mathrm{CH}_{3} \mathrm{CN}$ is then formed from the radiative association between the methyl ion and HCN (A.19), again followed by electron recombination (A.20):

$$
\begin{aligned}
& \mathrm{NH}_{3}+\mathrm{C}^{+} \rightarrow \mathrm{HCNH}^{+} \\
& \mathrm{HCNH}^{+}+\mathrm{e}^{-} \rightarrow \mathrm{HCN}+\mathrm{H} \text { or } \\
& \quad \mathrm{HCNH}^{+} \rightarrow \mathrm{HCN}+\mathrm{NH}_{4}^{+} \\
& \mathrm{CH}_{3}^{+}+\mathrm{HCN} \rightarrow \mathrm{CH}_{3} \mathrm{CNH}^{+}+h v \\
& \mathrm{CH}_{3} \mathrm{CNH}^{+}+\mathrm{e}^{-} \rightarrow \mathrm{CH}_{3} \mathrm{CN}+\mathrm{H} .
\end{aligned}
$$

Comparisons between the results from this model and observations suggest that a grain-surface formation of $\mathrm{CH}_{3} \mathrm{CN}$ is not required.

- On grain surfaces, $\mathrm{CH}_{3} \mathrm{CN}$ can be formed by successive hydrogenation of $\mathrm{C}_{2} \mathrm{~N}$ or by recombination between $\mathrm{CN}$ and $\mathrm{CH}_{3}$ :

- $\mathrm{C}_{2} \mathrm{~N} \stackrel{+\mathrm{H}}{\mathrm{HCCN}} \stackrel{+\mathrm{H}}{\longrightarrow} \mathrm{CH}_{2} \mathrm{CN} \stackrel{+\mathrm{H}}{\longrightarrow} \mathrm{CH}_{3} \mathrm{CN}$. This set of reactions, used in Caselli et al. (1993), underestimates the $\mathrm{CH}_{3} \mathrm{CN}$ abundance by a factor about 50 compared to the abundance observed in the Orion hot core. This could be explained by the fact that HCCN formation is in competition with $\mathrm{C}_{3} \mathrm{~N}$ formation, and the former is indicated by the authors as a less important pathway.

- $\mathrm{CN}+\mathrm{CH}_{3} \rightarrow \mathrm{CH}_{3} \mathrm{CN}$ (Hasegawa \& Herbst 1993), which yields a $\mathrm{CH}_{3} \mathrm{CN}$ abundance in good agreement with the Orion hot core value for an age of $10^{5} \mathrm{yr}$.

Regarding $\mathrm{C}_{2} \mathrm{H}_{5} \mathrm{CN}$, some studies point towards grain-surface formation of this molecule by hydrogenation of $\mathrm{HC}_{3} \mathrm{~N}$ (Blake et al. 1987; Charnley et al. 1992; Caselli et al. 1993). Observations by Liu \& Snyder (1999) are consistent with this theory. However, the abundance ratio of $\mathrm{CH}_{3} \mathrm{CN}$ to $\mathrm{C}_{2} \mathrm{H}_{5} \mathrm{CN}$ predicted by Caselli et al. (1993) is at least two orders of magnitude smaller than the ratio observed in hot cores and hot corinos. Therefore, it seems unlikely that both $\mathrm{CH}_{3} \mathrm{CN}$ and $\mathrm{C}_{2} \mathrm{H}_{5} \mathrm{CN}$ form via the surface reactions proposed by Caselli et al. (1993).

\section{A.5. Timescales for gas-phase processes}

For the molecules for which no strong argument has been proposed against gas-phase formation, e.g., $\mathrm{CH}_{3} \mathrm{OCH}_{3}$, we can estimate a formation timescale from the slowest rate coefficient of the reactions involved. In general, for a reaction between reactants $r_{1}$ and $r_{2}$ yielding a product $p$, we have:

$$
\frac{\mathrm{d} n_{\mathrm{p}}}{\mathrm{d} t}=k_{\mathrm{f}} n_{\mathrm{r}_{1}} n_{\mathrm{r}_{2}}
$$

where $k_{\mathrm{f}}$ is the reaction rate coefficient in $\mathrm{cm}^{3} \mathrm{~s}^{-1}$. In the event that $n_{\mathrm{r}_{1}} \gg n_{\mathrm{r}_{2}}$ (which is the case for $\mathrm{CH}_{3} \mathrm{OCH}_{3}$, as we will see further down), we also have $n_{\mathrm{r}_{1}}+n_{\mathrm{p}} \sim n_{\text {tot }}$, where $n_{\text {tot }}$ is defined as $n_{\mathrm{r}_{1}}+n_{\mathrm{r}_{2}}+n_{\mathrm{p}}$. Then,

$$
\begin{aligned}
\frac{\mathrm{d} n_{\mathrm{p}}}{\mathrm{d} t} & =k_{\mathrm{f}}\left(n_{\mathrm{tot}}-n_{\mathrm{p}}\right) n_{\mathrm{r}_{2}} \\
& =-k_{\mathrm{f}} n_{\mathrm{r}_{2}} n_{\mathrm{p}}+k_{\mathrm{f}} n_{\mathrm{tot}} n_{\mathrm{r}_{2}} \\
& =-\frac{1}{\tau_{\mathrm{f}}} n_{\mathrm{p}}+k_{\mathrm{f}} n_{\mathrm{tot}} n_{\mathrm{r}_{2}},
\end{aligned}
$$

which has solution of the form $n_{\mathrm{p}}(t)=C_{1} \exp \left[-t / \tau_{\mathrm{f}}\right]+C_{2}$, where $C_{1}$ and $C_{2}$ are constants. The formation timescale is

$$
\begin{aligned}
\tau_{\mathrm{f}} & =\left(k_{\mathrm{f}} n_{\mathrm{r}_{2}}\right)^{-1} \\
& =\left(k_{\mathrm{f}} n_{\mathrm{H}_{2}} x_{\mathrm{r}_{2}}\right)^{-1} \\
& =\left(\frac{k_{\mathrm{f}}}{10^{-10}}\right)^{-1}\left(\frac{n_{\mathrm{H}_{2}}}{10^{8}}\right)^{-1}\left(\frac{x_{\mathrm{r}_{2}}}{10^{-10}}\right)^{-1} 3 \times 10^{4} \mathrm{yr}
\end{aligned}
$$

where $n_{\mathrm{H}_{2}}$ is the $\mathrm{H}_{2}$ density in $\mathrm{cm}^{-3}$ and $x_{\mathrm{r}_{2}}$ is the abundance of $\mathrm{r}_{2}$, the least abundant reactant.

For $\mathrm{CH}_{3} \mathrm{OCH}_{3}$, the slowest reaction is (A.15), which has a reaction rate of $1 \times 10^{-10} \mathrm{~cm}^{3} \mathrm{~s}^{-1}$ (from the gas-phase chemical network osu.2005 ${ }^{3}$ ), and (predicted) $x_{\mathrm{r}_{2}}=x_{\mathrm{CH}_{3} \mathrm{OH}_{2}^{+}} \sim(1-5) \times$ $10^{-10}$ (from Rodgers \& Charnley 2003), which is much smaller than $x_{\mathrm{r}_{1}}=x_{\mathrm{CH}_{3} \mathrm{OCH}_{3}} \gtrsim 1 \times 10^{-8}$ (from Table 5), hence justifying the derivation and use of Eq. (A.27). For our sources, $n_{\mathrm{H}_{2}} \sim$ $10^{8}-10^{9} \mathrm{~cm}^{-3}$, which yields $\tau_{\mathrm{f}}=6 \times 10^{2}-3 \times 10^{4} \mathrm{yr}$.

Regarding the destruction of the studied species in the gasphase, we expect ions such as $\mathrm{H}_{3} \mathrm{O}^{+}, \mathrm{HCO}^{+}$, and $\mathrm{H}_{3}^{+}$to be the main destroyers of complex organic molecules during the hot corino phase, with $\mathrm{H}_{3} \mathrm{O}^{+}$being the most important of the three

\footnotetext{
3 http://www.physics.ohio-state.edu/ eric/ research.html
} 
due to the evaporation of water contained in the icy grain mantles (Ceccarelli et al. 1996; Rodgers \& Charnley 2003). In this case, we have:

$$
\begin{aligned}
\frac{\mathrm{d} n_{\mathrm{p}}}{\mathrm{d} t} & =-k_{\mathrm{d}} n_{\mathrm{p}} n_{\mathrm{H}_{3} \mathrm{O}^{+}} \\
& =-\frac{1}{\tau_{\mathrm{d}}} n_{\mathrm{p}} \\
\Rightarrow n_{\mathrm{p}}(t) & =C_{3} \exp \left(-t / \tau_{\mathrm{d}}\right),
\end{aligned}
$$

where $k_{\mathrm{d}}$ is the destruction rate, $\tau_{\mathrm{d}}$ is the destruction timescale, and $C_{3}$ is a constant. Then,

$$
\begin{aligned}
\tau_{\mathrm{d}} & =\left(k_{\mathrm{d}} n_{\mathrm{H}_{3} \mathrm{O}^{+}}\right)^{-1} \\
& =\left(k_{\mathrm{d}} n_{\mathrm{H}_{2}} x_{\mathrm{H}_{3} \mathrm{O}^{+}}\right)^{-1} \\
& =\left(\frac{k_{\mathrm{d}}}{10^{-10}}\right)^{-1}\left(\frac{n_{\mathrm{H}_{2}}}{10^{8}}\right)^{-1}\left(\frac{x_{\mathrm{H}_{3} \mathrm{O}^{+}}}{10^{-10}}\right)^{-1} 3 \times 10^{4} \mathrm{yr} .
\end{aligned}
$$

Destruction of $\mathrm{CH}_{3} \mathrm{OCH}_{3}$ by $\mathrm{H}_{3} \mathrm{O}^{+}$has $k_{\mathrm{d}}=10^{-9} \mathrm{~cm}^{3} \mathrm{~s}^{-1}$ (osu.2005) and $x_{\mathrm{H}_{3} \mathrm{O}^{+}}=7 \times 10^{-11}-5 \times 10^{-9}$ (from Ceccarelli et al. 1996 for the lowest value, and Rodgers \& Charnley 2003 and Stäuber et al. 2005 for the highest value), which yields $\tau_{\mathrm{d}}=6-4 \times 10^{3} \mathrm{yr}$. This is comparable to the mid-range of the formation scale. Moreover, as the protostar ages, its luminosity increases, causing the expansion of the evaporation front and the replenishment of the complex molecules, whether they are mantle species or whether they form from freshly evaporated $\mathrm{CH}_{3} \mathrm{OH}$ and/or $\mathrm{H}_{2} \mathrm{CO}$. Therefore, the destruction by $\mathrm{H}_{3} \mathrm{O}^{+}$ is likely compensated by the evaporation or formation of more complex molecules. 